\title{
Norwich Technologies' Advanced Low-Cost Receivers for Parabolic Troughs
}

\section{FINAL REPORT}

Project Title:

Project Period:

Project Budget:

Submission Date:

Recipient:

Address:

Award Number:

Project Team:

Contacts:

Email:
Advanced Low-Cost Receivers for Parabolic Troughs

10/01/2012-10/01/2013

$\$ 252,122$

December 28, 2013

Norwich Technologies Inc.

52 Bridge St.

White River Junction, VT 05001

DE-EE0005803

Creare, Inc.

ANSYS Consulting Group, Inc.

Thayer School of Engineering

Joel Stettenheim

President

Phone: 802-384-1333

stettenheim@norwitech.com 
DE-EE0005803

Advanced Low-Cost Receivers for Parabolic Troughs

Norwich Technologies

\section{EXECUTIVE SUMMARY}

This report summarizes the successful results of our SunShot project, Advanced Low-

Cost Receivers for Parabolic Troughs. With a limited budget of $\$ 251 \mathrm{~K}$ and in only 12 months, we have (1) developed validated optical and thermal models and completed rigorous optimization analysis to identify key performance characteristics as part of developing first-generation laboratory prototype designs, (2) built optical and thermal laboratory prototypes and test systems with associated innovative testing protocols, and (3) performed extensive statistically relevant testing. We have produced fully functioning optical and thermal prototypes and accurate, validated models shown to capture important underlying physical mechanisms. The test results from the first-generation prototype establish performance exceeding the FOA requirement of thermal efficiency $>90 \%$ for a CSP receiver while delivering an exit fluid temperature of $>650{ }^{\circ} \mathrm{C}$ and a cost $<\$ 150 / \mathrm{kW}_{\text {th }}$. Our vacuum-free SunTrap receiver design provides improvements over conventional vacuum-tube collectors, allowing dramatic reductions in thermal losses at high operating temperature as shown in Fig. A.

Fig. A. Dramatic improvement in thermal efficiency of secondgeneration commercial prototype of SunTrap receiver relative to the SOA vacuum tube PTR-70. Data plotted as a function of time of day for example summer day in Arizona. Time of day simulation includes effects such as insolation, mirror/receiver tilt, and cosine effects.

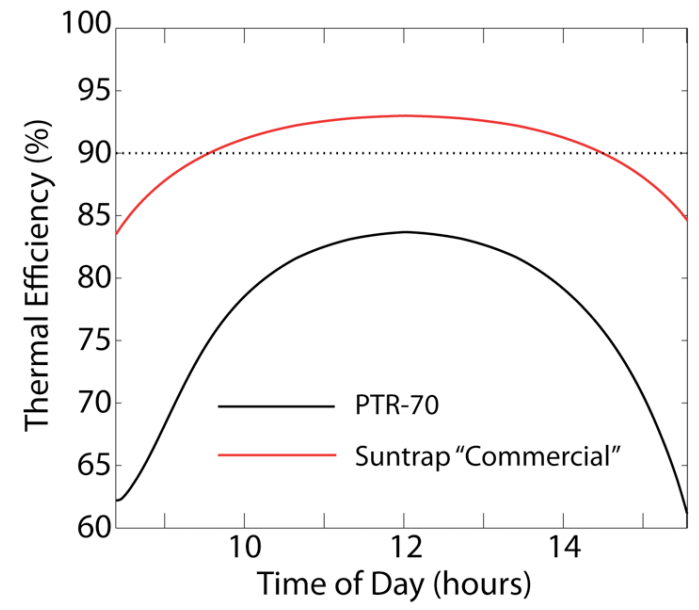

With follow-on funding, we are poised to dramatically impact CSP performance by building from the core of our demonstrated success. Given our proven ability to efficiently achieve significant results in a limited time frame, access to more substantial resources will result in commensurately larger returns. Our immediate development tasks are: (1) Fully optimize, design, build and test our second-generation prototype receiver. (2) Develop a low-cost, high-performance parabolic trough mirror collector leveraging the unique structural opportunities enabled by our receiver. (3) Continue our successful solar selective absorber work currently funded by an NSF SBIR.

Our successful project management approach is to leverage our technical and management expertise at Norwich Technologies (NT) to effectively coordinate the efforts of our team with our highly skilled sub-recipients, Creare and ANSYS. Central to NT's rapid technological progress and efficient use of resources is our strategy of using 
DE-EE0005803

Advanced Low-Cost Receivers for Parabolic Troughs

Norwich Technologies

external resources to quickly build highly sophisticated capabilities that are then transferred in-house for ongoing analysis and refinement. NT employees working on the project include PI Joel Stettenheim, PhD, CTO Troy McBride, PhD, Sr. R\&D engineer Oliver Brambles, PhD, R\&D engineer Emil Cashin, Project Manager Terry Donoghue, senior technician Ralph Golec, and technician Anders LaScala. Overall our team committed more than 4,000 hours to the project. Creare, Inc. personnel working on the project include Richard Kaszeta, PhD, Brynmor Davis, PhD, Nicholas Kattamis, PhD, Robert Payne, and Patrick Magari, PhD. Chi-Yang Cheng, PhD of ANSYS Consulting Group has provided primary expertise in the thermal analysis and use of the ANSYS Fluent CFD software tool. Six engineering students-Scott Snyder, Emil Cashin, Michelle Burns, Chloe Ruiz-Funes, Jeremy Broulliet, and Utkarsh Agarwal-together committed over 1,400 hours to the project through their two-term capstone engineering project course in Q1 and Q2. Through this mix of seasoned expertise and young engineering talent, we have completed the successful design, analysis, build, and testing of our novel receiver for parabolic-trough CSP systems with a limited budget and an accelerated schedule, generating significant value for the DOE. 
DE-EE0005803

Advanced Low-Cost Receivers for Parabolic Troughs

Table of Contents

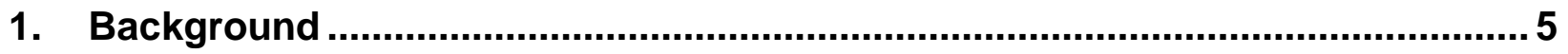

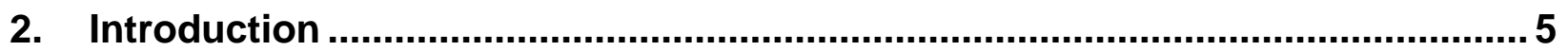

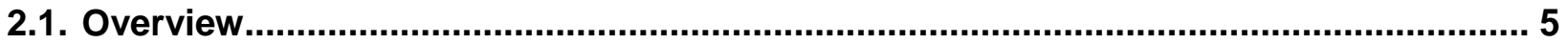

2.2. Statement of Project Objective (SOPO) Summary......................................................... 9

2.2.1 Phase 1: Prototype Analysis and Design (Months 0-6) .................................... 9

2.2.2 Phase 2: Prototype Build (Months 7-9) ...........................................................11

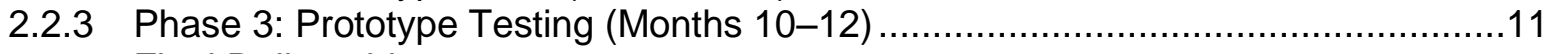

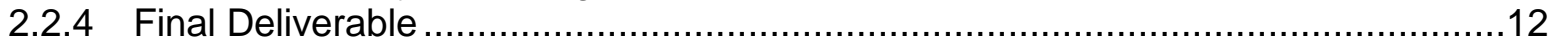

3. Project Results and Discussion................................................................ 12

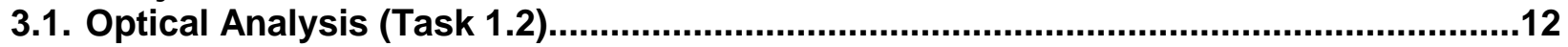



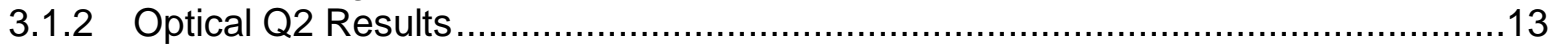

3.2. Thermal Analysis, Co-Optimization and System Considerations (Task 1.3) ...............13

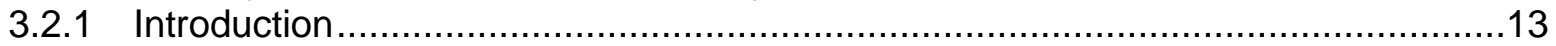

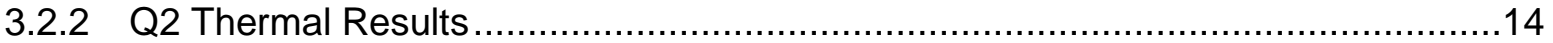

3.2.3 Co-optimization and Solar Field Performance .............................................14

3.2.4 Additional CFD Thermal and Fluid Modeling ..............................................16

3.3. Materials Selection, Cost and Performance Estimates and Final Specification (Tasks

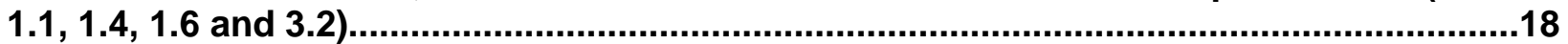

3.4. Thermal Test Protocols \& Apparatus, Thermal Prototype, and Material Selection

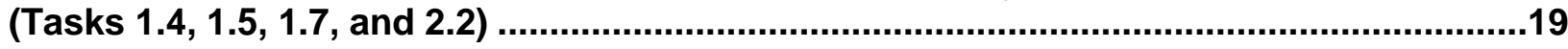

3.4.1 Thermal Test Protocols \& Apparatus ........................................................19

3.4.2 Thermal Test Design and Procedure .....................................................20

3.5. Optical Test Protocol \& Apparatus, Optical Prototype, and Materials Selection (Tasks

$1.4,1.5,1.7$, and 2.2)..............................................................................................................21

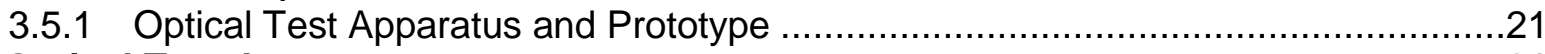

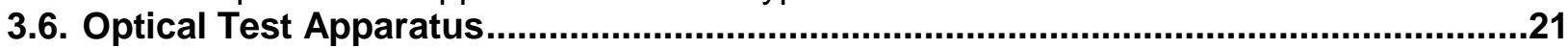

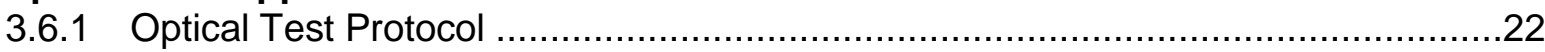

3.6.2 Optical Prototype Alignment...............................................................23

3.7. Optical and Thermal Testing, Model Refinement \& Validation, and Second-

Generation Commercial Prototype (Tasks 3.1 and 3.2) ....................................................24

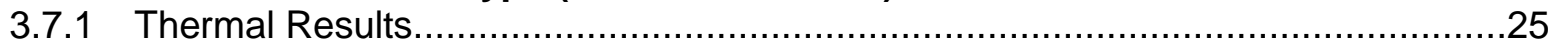

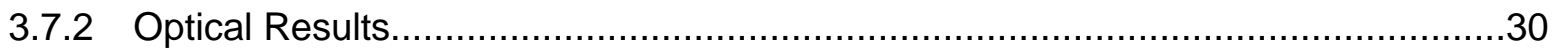



4. Conclusions........................................................................................... 35 
DE-EE0005803

Advanced Low-Cost Receivers for Parabolic Troughs

Norwich Technologies

\section{BACKGROUND}

Commercially available receivers for parabolic trough concentrating solar plants are vacuum-based heat collecting elements (HCEs). In these units, a central liquid-carrying tube with an outer optical-absorption coating is surrounded by a vacuum within a transparent concentric jacket. There are many challenges with such state-of-the-art vacuum receivers: e.g., their absorption coatings are expensive and technologically intensive, vacuum degradation causes failure of $1-5 \%$ of tubes per year, and the thick glass envelope is both expensive and fragile. i,i,iii $^{2}$ Thermal losses from such receivers are dominated by radiation; by the Stefan-Boltzmann law, radiative losses have a component that scales as $T^{4}$, challenging efficiency at high $T$.

As noted in the literature and the original FOA, to lower the levelized cost of energy (LCOE), a central drive in CSP is to increase temperature to improve operational plant efficiency and reduce storage costs. ${ }^{\mathrm{iv}, \mathrm{v}}$ As part of this effort despite their reduced high $T$ performance, HCE development for these plants has focused on the traditional vacuum tube architecture. For example, the Arcimedes HEMS11 receiver has the standard baffle, glass shell and vacuum structure. ${ }^{\text {vi }}$ Similarly the $4^{\text {th }}$ generation Schott receiver builds on previous designs with incremental improvements including an inner heat shield, reduced weight, protection caps at the ends, an updated but traditional multilayer cermet coating, and a modified bellows construction. ${ }^{\text {vii }}$

Burkholder's NREL 2008 test of Schott's PTR70 receiver demonstrates the reduced performance of traditional evacuated HCEs at high $T^{\text {viii }}$ Receiver heat losses are 225 $\mathrm{W} / \mathrm{m}$ at $400^{\circ} \mathrm{C}$, more than double to $475 \mathrm{~W} / \mathrm{m}$ at $500^{\circ} \mathrm{C}$, and rise dramatically to 1,248 $\mathrm{W} / \mathrm{m}$ at $650^{\circ} \mathrm{C}$ (fit extrapolation). As noted in Kutscher's 2010 Line Focus Study for NREL, ${ }^{\text {ix }}$ vacuum tube receivers heretofore have generally been considered by the CSP community as a mature technology with little opportunity for cost reductions or operational performance improvements

\section{INTRODUCTION}

\subsection{Overview}

The NT SunTrap parabolic trough receiver design (herein referred to as the SunTrap, the NT receiver, or simply "the receiver") offers dramatic reductions in heat loss over state-of-the-art receivers at high operating temperatures while avoiding the use and associated challenges of an evacuated tube. In particular, our receiver directly addresses the major challenges of vacuum based HCEs by (1) dramatically reducing radiation losses at higher temperatures, (2) significantly increasing reliability by 
DE-EE0005803

Advanced Low-Cost Receivers for Parabolic Troughs

Norwich Technologies

eliminating both vacuum and fragile all-glass tubes, (3) decreasing acquisition costs due to simpler structure and manufacture, and (4) operating at higher $T$.

Our highly successful 12-month project entailed extensive design modeling work, followed by the construction and testing of a prototype advanced receiver for troughbased CSP.

During the first two quarters of the project, we completed a rigorous overall design and analysis of our prototype receiver. Our approach included modeling the state-of-the-art existing receiver technology, validation of those models with literature data, and parameter analyses based on selected modifications to the state-of-the-art. This extensive modeling, combined with materials research including development of a large matrix of material options and characteristics, was used to refine and exhaustively characterize our novel receiver designs (Fig. 2).

Fig. 2. Left: CFD thermal models of SOA with no-vacuum. Right; ray-tracing analysis (edited to show only one ray, for simplicity) of incident raditaion, standard HCE.

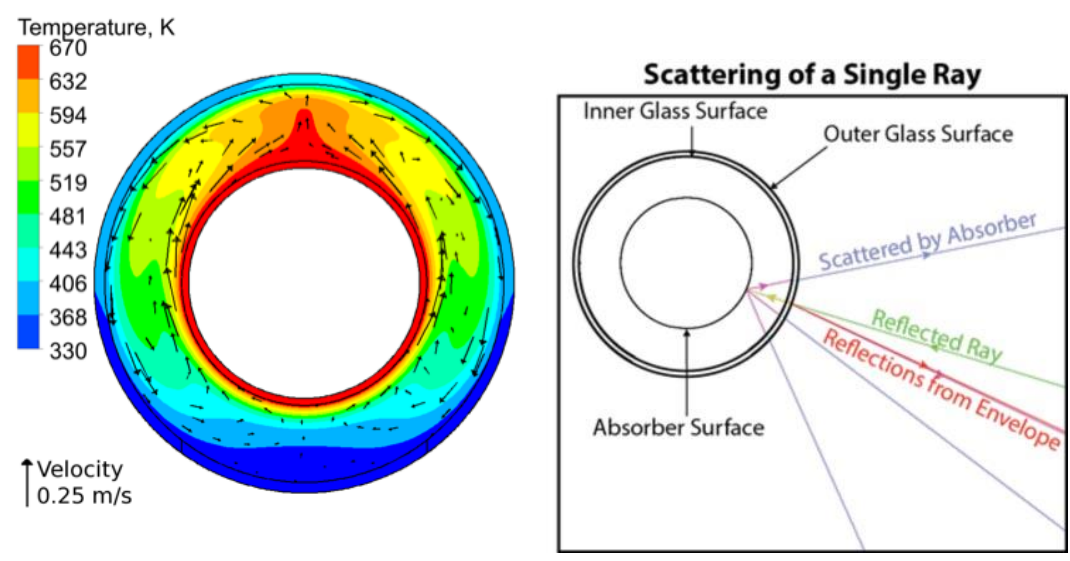

Thermal performance of candidate receiver configurations was determined by analyzing the results of radiative-loss, convective-loss, and conductive-loss models in ANSYS Fluent, a comprehensive finite-volume computational fluid dynamics (CFD) program. Radiative heat transfer losses were solved for using the discrete-ordinates radiation model, in which the spatial domain is discretized into a finite number of directions, and the discrete-ordinates model solves the radiative transfer equation for each discretized direction. The resources developed included 2D and 3D models with constant temperature and radiation flux boundary conditions, open domain models for wind effects, and pipe-flow models for heat flow to heat transfer fluid (HTF). The optical performance of the collector/receiver system was analyzed using Zemax and NT proprietary numerical analysis code. The powerful optical analysis resources developed included physical ray-tracing and numerical system optimization. Ray-tracing analysis allows for the quantification of a number of critical phenomena, including optical effects of coating reflectivity/absorbance, multiple reflections within the receiver, collector aberrations, receiver geometry, and overall receiver performance with respect to target specifications. Ray-tracing outputs, such as spatial irradiance profiles, were used as inputs to codes to model the detailed performance of the receiver in other tasks.

Efficient batch processing techniques were developed to enable parameter studies for the overall fine scale co-optimization of the thermal and optical performance. 
DE-EE0005803

Advanced Low-Cost Receivers for Parabolic Troughs

Norwich Technologies

Fig. 3 . Left: Co-optimized optical and thermal efficiency. Right: NT prototypes solar field thermal efficiencies versus PTR70 commercial unit as a function of incident solar energy.


During the third quarter we developed detailed fabrication level drawings for the receiver and the thermal and optical test facilities, fully specified the bill of materials, procured the relevant components, and began assembly.

The build and assembly was completed utilizing Creare's state-of-the-art facility in combination with outside services for simpler technical components. To reduce cost and increase speed, final assembly and on-going system refinements were carried out by NT technical staff. Materials development work included tests of the optical and mechanical properties of candidate coatings and structural components. Based on the resulting cost and performance data, we doubled our build to include two test geometries, significantly expanding the experimental parameter space we were able to explore during our test phase. We modified our test facility to evaluate both geometries.

Fig. 4. Optical test apparatus

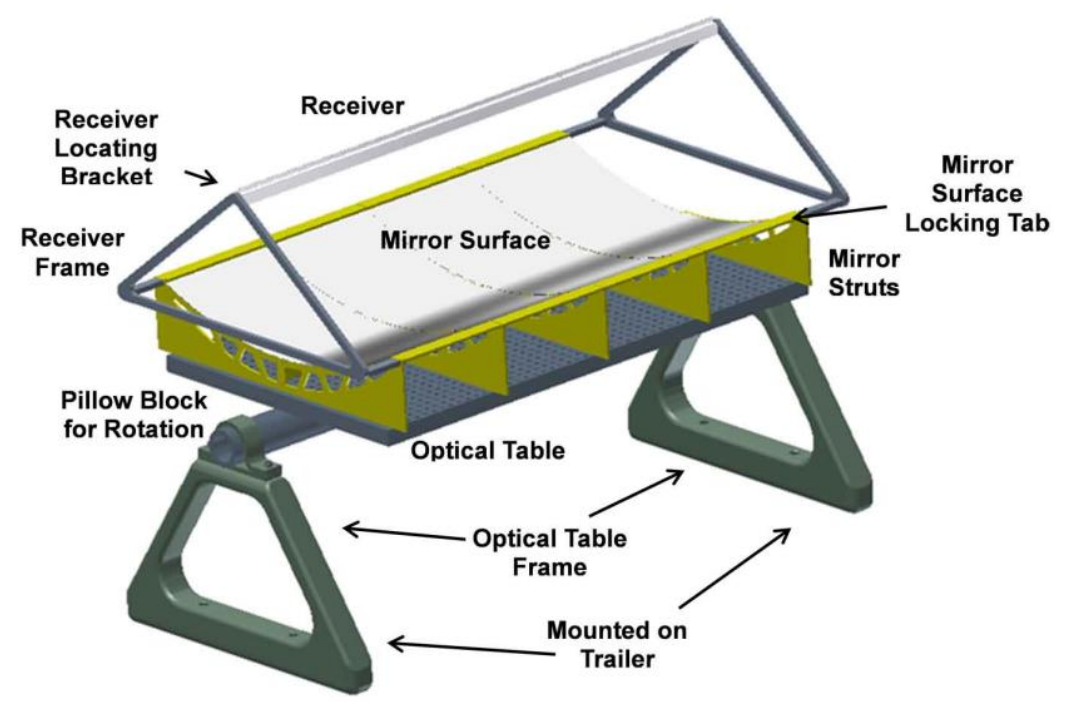

Development of thermal and optical test facilities (Fig. 4, Error! Not a valid bookmark self-reference.) included specifying test procedures. Our thermal test procedures 
DE-EE0005803

Advanced Low-Cost Receivers for Parabolic Troughs

Norwich Technologies

closely track the well-documented NREL procedures. For the optical test, we developed a new robust test using integration over a $~ 30$ minute period. The NREL "one-sun" optical test procedure has inherent imprecision; our optical test procedures refine the NREL approach with a more robust approach. Our simplified, integrated protocol eliminates the need for tracking; the article on our protocol that we are preparing for publication should be of interest to the CSP community.

Fig. 5. Left: Optical test stand, including collector on tilting base and optical prototype connected to fluid loop.

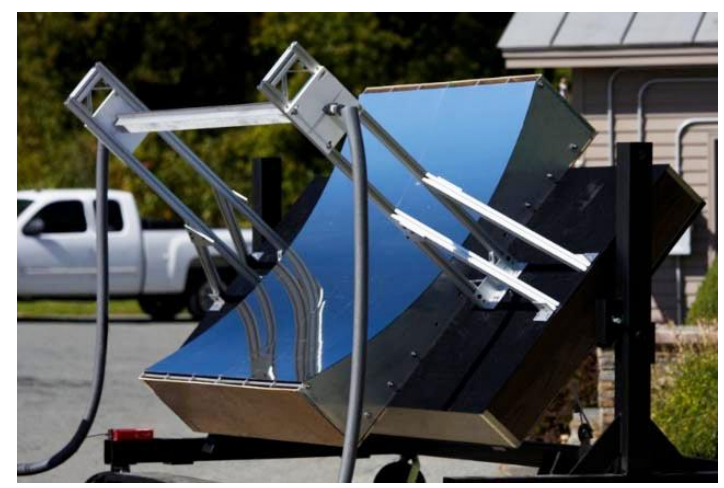

In the final quarter, we performed optical and thermal tests with the prototypes to refine and validate the thermal and optical models and to provide materials and performance operational feedback as part of developing our second generation commercial prototype. These tests were performed by NT engineers and scientists with support from Creare Inc. By leveraging effort efficiently, we were able to complete a rigorous design, build, and test cycle on a novel receiver technology within a 12-month period. Experimental results from our prototypes show reduction in heat loss over commercially available HCE technology at high temperature, with excellent agreement between design simulations and prototype measurements (Fig. 6).

Fig. 6. Left: Heat loss of NT first-generation and second-generation prototypes versus temperature showing dramatic improvement relative to SOA PTR70 vacuum tube. Right: Optical test normalized power profile for modeled (red) and measured data (blue) showing close correspondence
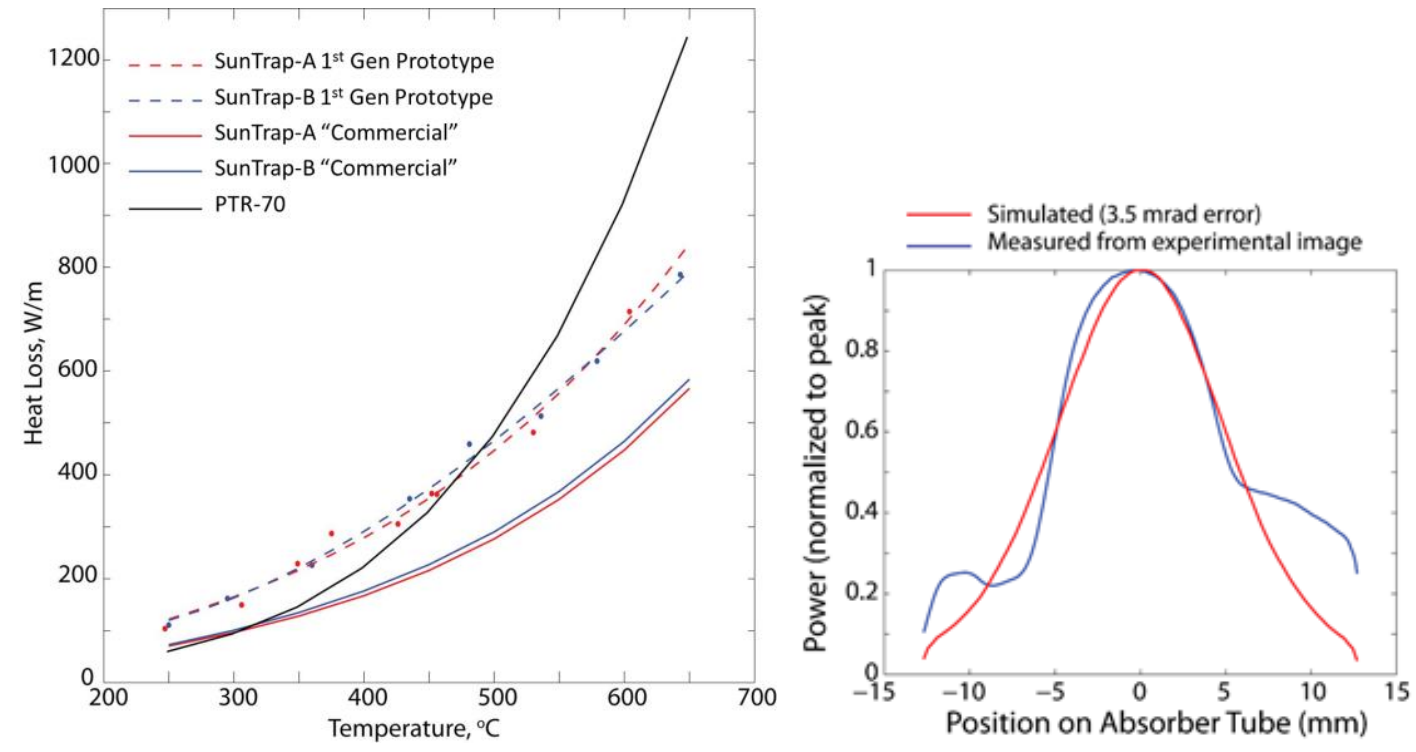

Page 8 of 36 
DE-EE0005803

Advanced Low-Cost Receivers for Parabolic Troughs

Norwich Technologies

\subsection{Statement of Project Objective (SOPO) Summary}

Our project milestones fall into a single 12-month budget period and into three Phases: (1) prototype analysis and design (months 0-6), (2) prototype build and test apparatus procurement and assembly (months 7-9), and (3) prototype testing and reporting (months 10-12).

\subsubsection{Phase 1: Prototype Analysis and Design (Months 0-6)}

Task 1.1: Definition of Figure of Merit for Optical and Thermal Properties of Available Materials. A survey of existing, commercially ready materials useful for prototype assembly will be conducted. A figure of merit for each material will be defined based on its optical performance and/or its thermal performance as well as unit cost. As an example, materials being considered as thermal insulators will be gauged according to the thermal conductivity multiplied by the cost per $\mathrm{kg}$ of material. Materials being considered for optical properties could be ranked according to their reflectivity multiplied by the cost per square meter. We will determine the most appropriate figure of merit for each subcomponent. Milestone: A correlation matrix designed for feed-in to the modeling efforts to analyze the cost/benefit ratio for various material combinations.

Task 1.2: Optical Analysis. We will use analytic and experimental tools to optimize the optical design of our advanced receiver. Ray-tracing software tools, among others, will be used to characterize a design grid of candidate materials and configurations. Raytracing analysis will allow the quantification of a number of critical phenomena, including the optical effects of coating reflectivity/absorbance, multiple reflections within the receiver, collector aberrations, receiver geometry, and overall receiver performance with respect to target specifications, étendue limits, etc. Ray-tracing outputs, such as spatial irradiance profiles, will be used as inputs to codes to model the detailed performance of the receiver in other tasks. Milestone: Specification of at least two geometric designs having a simulated optical absorbance $\geq 95 \%$ (i.e., exceeding the operational absorbance of state-of-the-art HCEs ). As used here, "optical absorbance" is the fraction of photons incident on the receiver hat is absorbed by the receiver. The major mechanisms of photon loss will be identified.

Task 1.3: Thermal Loss Analysis. Overall thermal performance of the candidate receiver configurations will be determined using radiative-loss, conduction-loss, and convectionloss models. Initial first-order analysis will be used to evaluate candidate designs. These first-order calculations will include estimates of conduction losses through the hightemperature insulation and through the aperture window and its edges. We will also analyze the impact of convection within the receiver, estimate the impact of candidate optical coatings on overall receiver performance, including allowance for the decomposition in performance of the optical coating with time. We will estimate the heat-transfer-fluid pressure drop to appropriately size the flow channels. The selected designs will be rigorously analyzed using a comprehensive finite-volume computational fluid dynamics (CFD) program. This CFD simulation will provide a complete view of the realistic operation of the system, including fluid-velocity and temperature fields for the receiver; overall thermal efficiency, wall heat flux, and temperature distributions; mass flow rate of fluid inside the tube or tubes; and additional physical parameters.

Consequently, CFD simulation will provide an operationally realistic determination of the overall thermal efficiency of our advanced receiver system based on specific 
DE-EE0005803

Advanced Low-Cost Receivers for Parabolic Troughs

Norwich Technologies

geometries, material properties and other design parameters. In addition, NT will do first-order calculations for flow rates and heating requirements for freeze prevention in the leading geometry candidates; modeling analysis will include a simplified Failure Modes and Effects Analysis to investigate any potential damage by a freeze event for the leading geometry. Milestone: Successful completion of thermal-loss analysis will result in specification of at least two receiver designs having simulated thermal efficiencies $\geq 90 \%$.

Task 1.4: Materials Selection. We will secure written price quotes for the leading materials identified in Tasks 1.1-1.3. For each candidate material, the most likely modes of failure will be identified and the mechanism of failure understood. The performance windows for each candidate material will be evaluated and defined using laboratory tests. In addition, using mass budgets based on candidate materials, NT will evaluate the receiver designs for weight and wind-loading. If necessary, design revisions will assure that weight and wind forces associated with the prototype receiver are compatible with existing support structures so that no (or minor) additional hardware is needed for retrofits. Milestone: This task will end with component selection, including specification of key materials for our prototype receiver. Written price quotes from vendors that supply each component will be obtained.

Task 1.5: Testing Procedure and Protocol. We will develop a formal testing procedure and protocol for the prototype receiver. Rigorous testing of optical, thermal, and materials performance will validate simulations. Unbiased analysis will be supported by definition of a test protocol prior to completion of the prototype. The protocol will include testing of thermal, optical, and materials performance of both a commercially available receiver as a control and the new prototype. Multiple measurements of each performance value will be made so that statistically relevant averages can be compared. Milestone: Procedures for optical and thermal prototype testing completed.

Task 1.6: Cost and Performance Estimates. During the prototype design process outlined above, cost and performance considerations will be characterized. The best designs selected from the first three tasks may not have the absolute highest predicted efficiency, but will have the best Figure of Merit (a combined optimum of high efficiency and low ultimate receiver cost to achieve lowest estimated LCOE). Included in ultimate receiver cost are manufacturability, durability, and ease of scaling to production quantities. For each candidate prototype design, a cost-and-performance estimate for the complete collector package will be performed. Primary indicators for a successful design are a predicted manufactured cost (per meter) less than state-of-the-art receivers and performance estimates that achieve the target values specified in the CSP R\&D FOA while supporting low maintenance costs. Milestone: Predicted manufactured cost of receiver along with the modeled performance is shown to be $\$ 150 / \mathrm{kW}_{\text {th. }}$.

Task 1.7: Finalized Prototype Design and Procurement. At the end of the 6-month prototype design process, a finalized prototype design and bill of materials will be generated. Ordering of all items for prototype build and equipment for prototype testing will be scheduled so as to complete the prototype build within 9 months from the start of this project. If there are indications of long lead-time items, NT will attempt to anticipate 
DE-EE0005803

Advanced Low-Cost Receivers for Parabolic Troughs

Norwich Technologies

such items and order prior to the 6-month point. A short report summarizing the final prototype design will be completed. Milestone: Interim report on final prototype design.

Go/No-Go Decision Point 1. A receiver for trough applications will be designed, modeled, and shown to operate at $\geq 90 \%$ thermal efficiency with an exit heat transfer fluid temperature $\geq 650{ }^{\circ} \mathrm{C}$ at a cost of $<\$ 150 / \mathrm{kW}_{\text {th }}$.

Patents and Publications. Patents for relevant intellectual property will be filed. Results of research published in high-visibility, high-impact, peer-reviewed journal(s). All publications resulting from the funded research will acknowledge that the work was supported by the U.S. DOE SunShot Initiative under the specific award number.

\subsubsection{Phase 2: Prototype Build (Months 7-9)}

This phase entails building of a prototype receiver. Construction of a test apparatus for the prototype will be commenced in this Phase and completed in Phase 3 with the procurement of long lead time equipment having been initiated in Phase 1.

Task 2.1: Company and Marketing Development. Throughout the project, NT will be working to build our company to prepare for the next phases of development. A part of this development will be market research and contacting of potential customers. Marketing information will be incorporated into the prototype design process, the performance requirements and associated testing procedures, and commercial prototype design process. Likewise, information from those studies will used in marketing development. At the end of the project period, Norwich Technologies will be poised to produce a commercial prototype receiver that can be field installed and dropin replaced with existing parabolic mirror installations. Milestone: Demonstration partner identified for commercial prototype receiver field testing and a letter of commitment is signed and in place.

Task 2.2: Build Prototype; Start Build of Test Apparatus. Prototype receiver will be fully assembled within 9 months of the start of the project. Construction of the test apparatus as detailed in the test procedure from Task 1.5 will be initiated. During first prototype fabrication, considerations and techniques for ultimate low-cost manufacturing will be evaluated and recorded. NT will supervise and direct the prototype fabrication to be completed by a subcontractor. Milestone: Prototype receiver assembly completed, all quality checks have passed, and the unit has passed commissioning tests.

\subsubsection{Phase 3: Prototype Testing (Months 10-12)}

This phase entails testing of our prototype and final reporting.

Task 3.1: Test Prototype. Testing of the prototype receiver will characterize performance in the three key areas of (1) optical efficiency, (2) thermal efficiency, and (3) material performance. In all instances, results will be compared with expected results from modeling. In cases where the results deviate substantially $(>5 \%)$ from the model behavior, analysis will be performed to either improve the modeling or remedy the discrepancy. In this manner, we expect to produce not only a tested prototype, but validated modeling tools for the prototype receiver design. This will allow for optimization not only in the prototype design but refinement of the predictive accuracy of the modeling tools. Milestone: Submit paper to a peer-reviewed scientific journal detailing the optical and thermal test results. Optical and thermal performance numbers will be itemized and compared with expected results and to the commercial standard. 
DE-EE0005803

Advanced Low-Cost Receivers for Parabolic Troughs

Norwich Technologies

Task 3.2: Project Management and Reporting. Reports and other deliverables will be provided in accordance with the Federal Assistance Reporting Checklist following the instructions included therein. Milestone: Final report, including commercial prototype geometric and material design.

2.2.4 Final Deliverable

A functioning prototype HCE for trough-type CSP thoroughly tested for optical and thermal efficiency and demonstrated to perform at $\geq 90 \%$ thermal efficiency while delivering a heat transfer fluid with an exit temperature of $\geq 650^{\circ} \mathrm{C}$, as demonstrated by testing performed under Task 3.1 based on the procedures and protocols defined under Task 1.5. Validated optical and thermal 1-d lumped parameter and 2-d \& 3-d CFD models for prototype receiver. Projected (itemized, defensible estimate) bulkmanufacturing cost of receiver will be $\leq \$ 150 / \mathrm{kW}_{\text {th }}$.

\section{PROJECT RESULTS AND DISCUSSION}

Given the limited space available in this summary report, discussion of related work under separate tasks has been combined. This approach has the additional advantage of providing a more coherent and less disjointed discussion of the work performed and the progress achieved.

\subsection{Optical Analysis (Task 1.2)}

\subsubsection{Optical Background}

In Q1 of this project, Zemax optical models were developed with collaboration with Brynmor Davis, PhD of Creare. Several thousand optical cases were explored in Q1 through the use of macros and the work of Thayer School of Engineering students and NT employees. Key insights from Q1 work were:

- Aim of the optical design for our receiver is to match SOA optical performance and increase efficiency by improving thermal performance.

- Improvements (decreases) in collector error have a greater impact on optimal efficiency of the candidate receivers than SOA.

Initial co-optimization with thermal modeling led to down-selection of conceptual alternatives at the end of Q1 to an optimized receiver geometry. We investigated the possible use of a secondary reflector within the receiver; this idea was explored during Q2 before being abandoned due to practical considerations (i.e., expected cost and performance of required high temperature high reflectivity material). 
DE-EE0005803

Advanced Low-Cost Receivers for Parabolic Troughs

Norwich Technologies

\subsubsection{Optical Q2 Results}

Most Q2 optical work consisted of running thousands of optical cases to compare the optical efficiency of the most promising geometries. The results were used with the thermal studies for cooptimization of the receiver for various operating scenarios. Optical efficiencies for a geometry with secondary reflector and our SunTrap geometry (no secondary reflector) are shown in Fig. 7. Assumptions include that the

Fig. 7. Plot of optical efficiency as a function of absorber width and collector focal length for a secondary reflector geometry (left) and our SunTrap geometry (no-secondary reflector) (right).
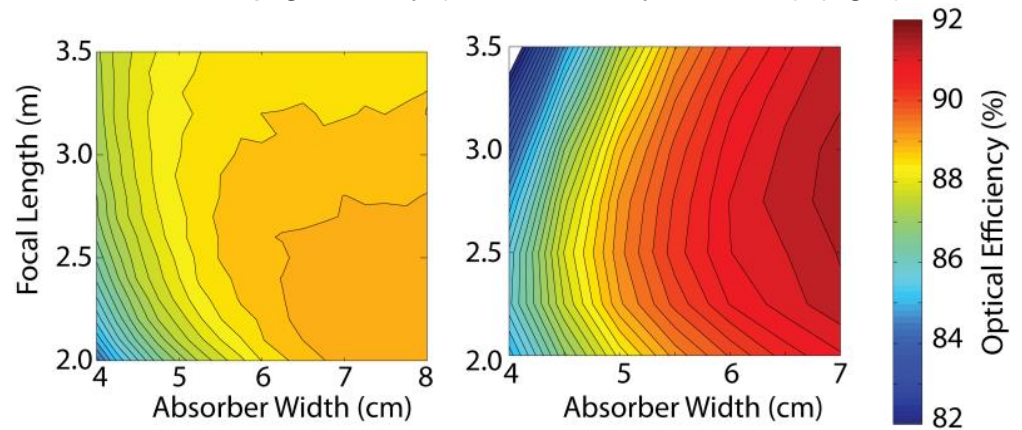
secondary reflector has $95 \%$ reflectivity, mirror diameter is $5 \mathrm{~m}$, and optical error is 3 mrad. With a near ideal reflectivity of $95 \%$, the optical efficiency of the receiver with secondary reflector is much lower except at smaller absorber sizes and longer focal lengths. In short, secondary reflector design offers increased thermal performance at the cost of reduced optical efficiency.

By performing thousands of Zemax optical simulations, we studied a wide parameter space for our receiver. Co-optimization with thermal results is presented in Sec. 3.2.3.

\subsection{Thermal Analysis, Co-Optimization and System Considerations (Task 1.3)}

\subsubsection{Introduction}

In Q1 and Q2 in conjunction with Dr. Chi-Yang Cheng of Ansys Consulting Group, the thermal performance of candidate receiver configurations (e.g., Fig. 8) was determined by analyzing the results of radiative-loss, convective-loss, and conductive-loss models in ANSYS FLUENT, a comprehensive finite-volume CFD program. Radiative heat transfer losses were solved for using the discrete-ordinates radiation model, in which the spatial domain is discretized into a finite number of directions, and the discreteordinates model solves the Fig. 8. Plot of thermal efficiency as a function of absorber width and focal length for two geometries - receiver with secondary reflector (Left) and SunTrap geometry (Right), as well as for 550 and $650 \stackrel{\circ}{\circ}$ operation. For the SunTrap case, the insulation thickness and focal length are chosen at a level which produces maximum total performance.
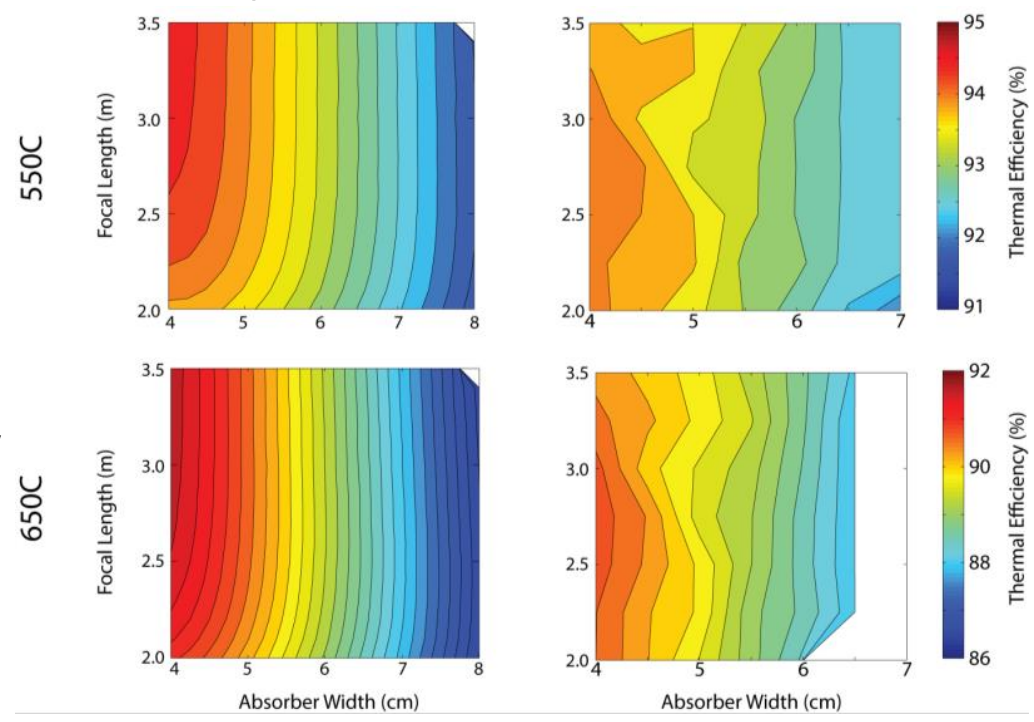

radiative transfer equation for each discretized direction. The radiation, conduction, and convective heat-transfer models are coupled in the FLUENT solver through the energy 
DE-EE0005803

Advanced Low-Cost Receivers for Parabolic Troughs

Norwich Technologies

equation and the core Navier-Stokes equations. FLUENT can capture the effects of material properties such as surface emissivity and temperature-dependent gas behavior.

The resulting thermal models are based on a two-dimensional cross section of the receiver with a constant-temperature boundary condition at the external surface of the absorber tube. This boundary condition reflects parabolic trough plant operation for a target heat transfer fluid loop outlet temperature. A model was developed to satisfy the general methodology of Khalsa and $\mathrm{Ho}^{\mathrm{x}}$ as stipulated in Task 1.3. This model was driven by applying an appropriate distribution of incident radiation to the receiver aperture. It proved computationally unwieldy, however, and did not accurately account for total energy flux, so we opted to continue using Zemax ray-tracing software to assess optical performance. The Khalsa and Ho method is appropriate for power tower fields in which repeated ray-tracing is undesirable; for our much simpler geometry, it is undesirable to add computational time and inaccuracy to thermal simulations. The radiation profile on the absorber tube from the Zemax ray-tracing software can be directly used as an input boundary condition on the coupled 3-D pipe flow/heat loss model.

\subsubsection{Q2 Thermal Results}

To characterize the performance space of our conceptual geometries, we conducted a series of geometric parameter studies. We developed in-house capability to do "batch" runs of thermal CFD simulations for parameter studies, varying quantities such as temperature, absorber width, secondary reflector vs. no secondary reflector, insulation depth, insulation thickness, and tilt angle. Several hundred thermal cases were explored to complement our thousands of optical cases. These sensitivity analyses gave insight into the overall (optical + thermal) behavior of different receiver geometries and allowed the development of a co-optimized receiver design based on both optical and thermal performance.

By performing hundreds of ANSYS Fluent CFD thermal simulations, a wide parameter space for our receiver geometries was studied. Co-optimization with optical results is presented in the following section.

\subsubsection{Co-optimization and Solar Field Performance}

Much of the Q2 simulation work was focused on co-optimization of the thermal and optical performances (Fig. 7, Fig. 8) of the receiver for different operating scenarios. Additionally, a model of the solar field operating from an inlet to outlet temperature was developed. The solar field model allows us to simulate plant performance and compare these results with the SOA for different operating scenarios.

To begin co-optimization, thermal and optical data was generated for a wide parameter space. For the no-CPC case, additional parameters must be optimized, as thermal performance depends on the chosen insulation depth and insulation thickness. Thermal performance data must therefore be developed iteratively with optical performance to find the best overall performance. 
DE-EE0005803

Advanced Low-Cost Receivers for Parabolic Troughs

Norwich Technologies

For each case, a best SunTrap design has been selected. That is, for the thermal data in each SunTrap case, insulation thickness and insulation depth (which do not affect optical performance) are chosen to produce maximum thermal efficiency (Fig. 8).

Combining thermal and optical results, a plot of overall receiver efficiency can be developed. The SunTrap geometry with no secondary reflector overall performance is slightly higher than the secondary reflector geometry performance. Overall, the secondary reflector solution has better thermal performance than the design without secondary reflector but worse optical performance due to imperfect reflectivity of the secondary reflector surface (modeled as $95 \%$ as a best case). At lower temperatures, optical performance is more important as heat loss is lower. Up to $650{ }^{\circ} \mathrm{C}$, the receiver

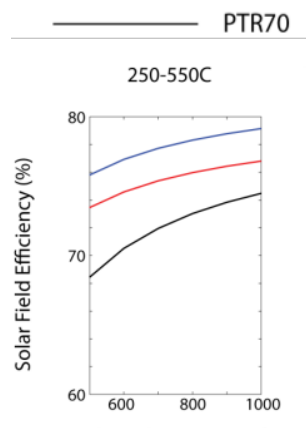

Incident Solar Energy $\left(\mathrm{W} / \mathrm{m}^{2}\right)$

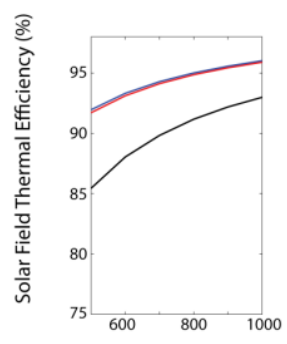

Incident Solar Energy $\left(\mathrm{W} / \mathrm{m}^{2}\right)$



Incident Solar Energy $\left(\mathrm{W} / \mathrm{m}^{2}\right)$

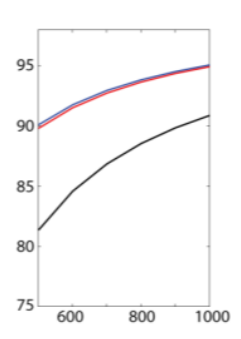

NT (no shading)
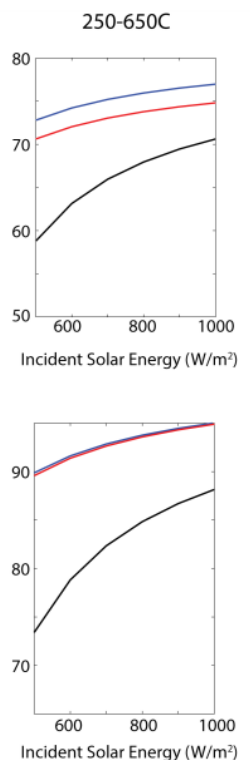

NT (shading)

$350-650 \mathrm{C}$
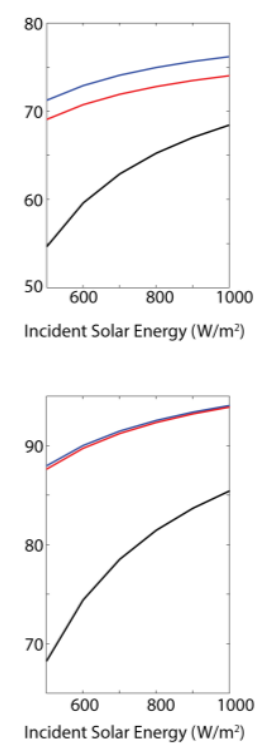

Fig. 9. Plot of solar field overall efficiency (top row) and thermal efficiency only (bottom row) as a function of incident solar energy (insolation) for various inlet and outlet solar field operating temperatures. In all cases, the NT collector outperforms the SOA Schott PTR70 receiver. The results are shown for the no-CPC design and shown with and without shading to highlight the further cost optimization that would determine whether to include a penalty for a wider mirror gap.

without secondary optics performs better than a design with a near-ideal secondary reflector. Given the reduced cost and complexity of the no secondary reflector design, NT selected that design for prototype construction.

NT developed a solar field model in MATLAB, allowing us to study the simulated plant performance over modeled operating conditions (Fig. 9). Operating conditions include the collector run temperature profile from inlet to outlet temperature, the variable insolation during a typical day, and tilt angle of the receiver at different times of the day. As shown in Fig. A (Executive Summary), the NT receiver substantially outperforms the SOA receiver for a simulated operating day. Indeed, the NT receiver has similar operating performance at current $400{ }^{\circ} \mathrm{C}$ solar field outlet operating temperatures, and outperforms the SOA receiver (while also eliminating vacuum) at all elevated operating temperatures (Fig. 9). Because the NT receiver has significantly reduced thermal losses as compared with SOA receiver at elevated temperature, the overall performance of the receiver improves relative to the SOA receiver at low insolation, as shown in Fig. 3.

In summary, rigorous thermal and optical modeling has allowed the co-optimization of the NT receiver design. The NT design substantially outperforms the SOA receiver at elevated operating temperatures, with benefits increasing at increasing operating 
DE-EE0005803

Advanced Low-Cost Receivers for Parabolic Troughs

Norwich Technologies

temperature. The resulting models and methods enable us to generate an optimized receiver geometry for a given operating temperature and collector. Near-optimal geometries were selected for prototyping based on standard stock tubing sizes.

\subsubsection{Additional CFD Thermal and Fluid Modeling}

Due to the heat-loss reductions of the NT design, our efficiency advantage is actually increased when maximum absorber temperatures are considered, despite a greater $\Delta T$ required for un-enhanced heat transfer. For example, in order to achieve an exit temperature of $650^{\circ} \mathrm{C}$, the SOA system must run to a pipe temperature of $665-670{ }^{\circ} \mathrm{C}$ as compared to $680^{\circ} \mathrm{C}$ for the NT receiver. The increase in heat loss for the SOA receiver is $116 \mathrm{~W} / \mathrm{m}$ from $650^{\circ} \mathrm{C}$ to $670{ }^{\circ} \mathrm{C}$, compared to an increase of $66 \mathrm{~W} / \mathrm{m}$ from $650^{\circ} \mathrm{C}$ to $680^{\circ} \mathrm{C}$ for the NT receiver.

For the solar field as a whole, the NT receiver requires absorber temperatures of 380 $680^{\circ} \mathrm{C}$ in order to heat the fluid from $350^{\circ} \mathrm{C}$ to $650^{\circ} \mathrm{C}$. In this case, the thermal efficiency of the solar field at a solar flux of $1000 \mathrm{~W} / \mathrm{m}^{2}$ decreases from $94.1 \%$ to $93.2 \%$. In comparison, the thermal efficiency of the PTR70 running from $370-670{ }^{\circ} \mathrm{C}$ would be reduced from $85.4 \%$ to $83.3 \%$ (and similar reductions for the Archimede HEMS11). At lower values of solar flux, the heat transfer and required $\Delta T$ is reduced, resulting in reduced impacts on efficiency.

At this time, two SunTrap geometries with different receiver tubes were again considered - SunTrap-A and SunTrap-B. Both geometries were modelled to analyze the trade-offs for each design.

To assess the performance of each geometry, a coupled 3D pipe flow/heat loss FLUENT model was developed. The model assumed that the fluid was Solar Salt at a temperature of $550^{\circ} \mathrm{C}$ with a mass flow rate of $6.22 \mathrm{~kg} / \mathrm{s}$ (taken from ENEA 2001). ${ }^{\mathrm{xi}}$ In each pipe flow simulation, it was assumed that $4000 \mathrm{~W} / \mathrm{m}$ of radiation was uniformly incident along the exposed surface of the pipe. For all simulated geometries, the average temperature of the absorber tube along the insulated section was the same as the fluid temperature (to within $+/-1 \mathrm{~K}$ ). However, the average temperature of the exposed section increases as the pipe diameter increases, as shown in Fig. 10. For SunTrap-A with a tube of width $5.5 \mathrm{~cm}$ has less effective heat transfer (higher $\Delta T$ ) than SunTrap-B pipes with diameters of less than 7.5 $\mathrm{cm}$. The poorer relative performance of the SunTrap-A design in terms of heat transfer efficiency to the fluid is due to locally low heat-transfer coefficients in Fig. 10 . Variation of $\Delta T$ (between fluid and absorbing surface) with varying SunTrap-B tube diameter. For comparison, $\Delta T$ for SunTrap-B is included in red.

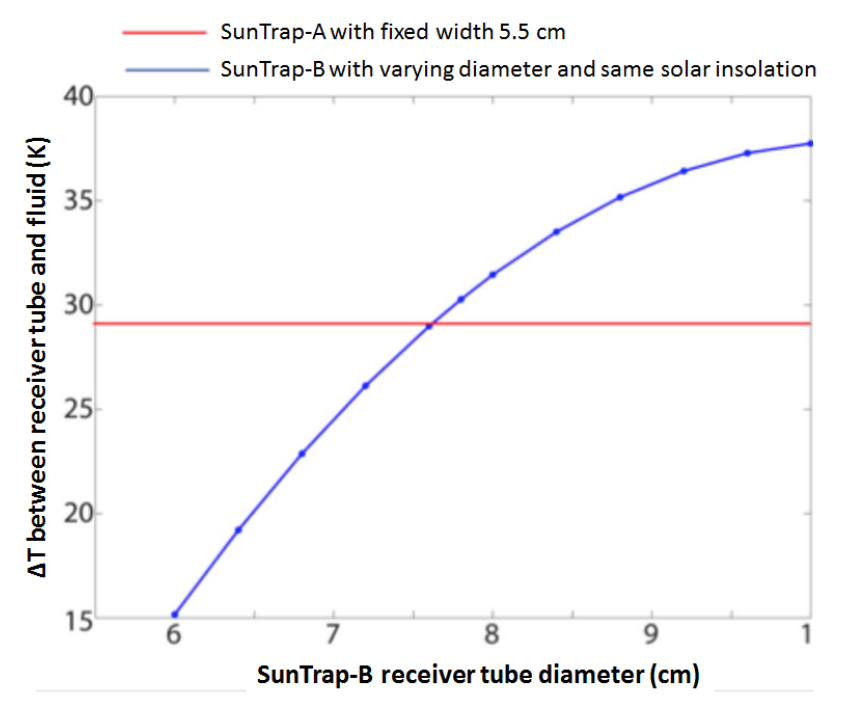

slow-flowing regions. 
DE-EE0005803

Advanced Low-Cost Receivers for Parabolic Troughs

Norwich Technologies

The dramatic rise in $\Delta T$ as the cylinder increases in diameter $d$ is due to the decrease in the heat transfer coefficient between the fluid and the inner pipe surface. For the same mass flow rate, flow velocity is proportional to $1 / d^{2}$ and the Reynolds number is proportional to $1 / d$. The reduced Reynolds number of the flow at larger tube diameters acts to reduce the heat transfer coefficient, resulting in increased $\Delta T$.

Table 1 shows how tube diameter influences heat loss at $400^{\circ} \mathrm{C}, 550^{\circ} \mathrm{C}$, and $650^{\circ} \mathrm{C}$. The optimum circular tube diameter for minimizing heat loss for SunTrap-B at higher $T$ is $\sim 7.2 \mathrm{~cm}$ (minima are highlighted). As diameter is increased, exposed radiating surface area decreases, reducing heat loss, but the difference in temperature between HTF and absorber tube is raised (as shown in Fig. 10 ), resulting in higher radiating surface temperature and increased heat loss. Therefore, a compromise must be sought between these two competing factors. SunTrap-A outperforms the optimized SunTrap-B by $25 \mathrm{~W} / \mathrm{m}$ at $400^{\circ} \mathrm{C}, 48 \mathrm{~W} / \mathrm{m}$ at $550^{\circ} \mathrm{C}$, and $71 \mathrm{~W} / \mathrm{m}$ at $650^{\circ} \mathrm{C}$.

Table 1. Variation of Heat Loss with varying SunTrap-B receiver tube diameter with minima highlighted. SunTrap-A provided at end of table for comparison.

\begin{tabular}{|c|c|c|c|}
\hline $\begin{array}{c}\text { SunTrap-B } \\
\text { Tube Diam }(\mathrm{cm})\end{array}$ & Heat Loss, $400^{\circ} \mathrm{C}(\mathrm{W} / \mathrm{m})$ & Heat Loss, $550^{\circ} \mathrm{C}(\mathrm{W} / \mathrm{m})$ & Heat Loss, $650^{\circ} \mathrm{C}(\mathrm{W} / \mathrm{m})$ \\
\hline 6 & 178 & 377 & 609 \\
\hline 6.4 & 177 & 371 & 597 \\
\hline 6.8 & 176 & 370 & 593 \\
\hline 7.2 & 177 & 370 & 592 \\
\hline 7.6 & 181 & 374 & 596 \\
\hline 8 & 183 & 377 & 599 \\
\hline 8.4 & 184 & 381 & 603 \\
\hline 8.8 & 186 & 384 & 606 \\
\hline 9.2 & 189 & 387 & 610 \\
\hline 9.6 & 191 & 389 & 612 \\
\hline 10 & 193 & 392 & 615 \\
\hline $\begin{array}{c}\text { SunTrap-A } \\
(5.5 \mathrm{~cm})\end{array}$ & 154 & 323 & 523 \\
\hline
\end{tabular}

To assess the fluid flow advantages of SunTrap-B relative to SunTrap-A, finite-element analyses were performed to examine the stresses acting on the absorber pipe due to the fluid pressure. In this model, it was assumed that the fluid pressure acting on the interior of the pipe was 5 bar as used in the initial design of the Archimede plant by ENEA $^{\text {xii }}$ and the yield stress of the Inconel 625 used for the absorber tube was 345 $\mathrm{MPa}$. For a SunTrap-B tube of thickness of $3 \mathrm{~mm}$ and diameter of $72 \mathrm{~mm}$, the maximum stress acting on the pipe was $6.2 \mathrm{MPa}$, giving a factor of safety of 55 . In contrast, for a SunTrap-A tube of thickness $3 \mathrm{~mm}$, diameter $60 \mathrm{~mm}$, the localized maximum stress acting on the pipe is $41 \mathrm{MPa}$ resulting in a factor of safety of 8.4. When the absorber tube is heated, the yield stress will decrease by approximately $50 \%$, which will further reduce this factor of safety to possibly unacceptable margins.

The head loss in the $5.5 \mathrm{~cm}$ SunTrap-A tube using molten salt is estimated to be approximately $20 \%$ lower than the losses in the current oil HTF systems. However, the head loss for the $7.2 \mathrm{~cm}$ SunTrap-B tube using molten salt is $60 \%$ lower than the SunTrap-A $5.5 \mathrm{~cm}$ tube using molten salt. It is therefore necessary to use a larger operating pressure (and corresponding pumping power) for SunTrap-A, which may make the use of the SunTrap-B more appealing from a plant operation standpoint. 
DE-EE0005803

Advanced Low-Cost Receivers for Parabolic Troughs

Norwich Technologies

These analyses suggest that there are potential performance advantages for SunTrap-A and potential operational advantages for the SunTrap-B. This investigation was performed concurrently with the conceptual stages of prototype design, in which it became clear that prototyping both SunTrap-A and SunTrap-B receiver geometries was reasonable for the project scope and schedule. Developing dual prototype geometries offered us both the ability to better quantify their performance differences and a second validation case for our models.

\subsection{Materials Selection, Cost and Performance Estimates and Final Specification (Tasks 1.1, 1.4, 1.6 and 3.2)}

Based on our test results and validated models, a commercial-scale receiver design can be optimized as part of a receiver-collector system. Receiver geometry (absorber size, acceptance angle, etc.) varies based on collector aperture and accuracy. For a given collector aperture and accuracy (or a range thereof), an optimal combination of receiver focal length, acceptance angle, and absorber tube size can be generated using the optical/thermal co-optimization method we have developed. This analysis will be performed in the future during co-development with a collector manufacturer.

As shown in Table 2, the projected cost for our receiver design is approximately half of the FOA target of $\$ 150 / \mathrm{kW}$ of heat delivered. Our receiver is able to efficiently operate at higher temperature with a cost that is less than or comparable to current parabolic trough HCEs. Our goal is to offer higher performance than current receivers at a lower cost per receiver and with reduced maintenance requirements; this is a readily attainable goal with manufacturing efficiencies.

Table 2. Estimated mass-manufacturing cost breakdown for commercial NT SunTrap receiver. A dominant cost component is the absorber tube. A near-term design optimized for operation with a 316-family absorber could compete on cost with SOA receiver designs while offering higher performance.

\begin{tabular}{|c|c|c|c|}
\hline Component & Material & Cost for $4 \mathrm{~m}$ Section & Justification \\
\hline Absorber Tube & 316 SS or Inconel & $\begin{array}{l}\text { 316SS: } \$ 300 \\
\text { Inconel: } \$ 600\end{array}$ & $\begin{array}{l}\text { Est. from London Metals } \\
\text { Exchange pricing }\end{array}$ \\
\hline Coating & $\begin{array}{c}\text { Atmospherically } \\
\text { Stable Solar Selective } \\
\text { Coating }\end{array}$ & $\$ 20$ & Internal estimate \\
\hline Glass Cover & AR-coated Borofloat & $\$ 100$ & Prototyping exp. \\
\hline Other & & $\$ 280$ & \\
\hline Steel Shell & 304 SS & $\$ 100$ & Prototyping exp. \\
\hline Mfg. and other & & $\begin{array}{l}550^{\circ} \mathrm{C}: \$ 80 \\
650^{\circ} \mathrm{C}: \$ 110\end{array}$ & $10 \%$ \\
\hline $\begin{array}{l}\text { Total Cost for } \\
4 \mathrm{~m} \text { Receiver }\end{array}$ & & $\begin{array}{c}\text { With 316SS : } \$ 880 \pm 20 \% \\
\text { With Inconel : } \$ 1210 \pm 25 \%\end{array}$ & $\begin{array}{l}\text { Cost/Capacity: } \\
\$ 65-\$ 80 / \mathrm{kW}_{\text {th }}\end{array}$ \\
\hline
\end{tabular}

Cost uncertainty is primarily driven by the selection of an absorber tube material. Current salt trough receivers use $316 \mathrm{Ti}^{\text {xiii }}$ for $550^{\circ} \mathrm{C}$ operation; Solar Two development indicates that Inconel is suitable for $650^{\circ} \mathrm{C}$ operation and that various stainless steel compositions may be acceptable as well. ${ }^{\text {iv }}$ Cost spread for Inconel and SS options is presented in Table 2; Inconel is substantially more expensive than steels due to its high nickel content. Ultimately, absorber material selection for $650^{\circ} \mathrm{C}$ operation will be driven by an extensive testing program with compatible HTF compositions. 
DE-EE0005803

Advanced Low-Cost Receivers for Parabolic Troughs

Norwich Technologies

Reducing failure and maintenance costs is emphasized by NREL. ${ }^{x v}$ Conventional tube failure due to vacuum loss and breakage is unacceptably high: Kutscher et al. (2010) state, "the most recent data for SEGS indicated that receiver tube failures had decreased to $3.37 \%$ of the total field receivers per year. Of these failures, $55 \%$ were reported to involve broken glass and $29 \%$ involved loss of vacuum, in most cases due to the failure of glass/metal seals, but also due to bowing tubes ... A receiver tube breakage rate of $3.4 \%$ per year seems unacceptably high, given that the receivers themselves represent $30 \%$ of the solar field material cost and would require additional labor to replace." Eliminating vacuum and replacing most of the glass with a robust metal receiver shell (as opposed to a self-supporting glass tube) should slash O\&M costs for the solar field.

\subsection{Thermal Test Protocols \& Apparatus, Thermal Prototype, and Material Selection (Tasks 1.4, 1.5, 1.7, and 2.2)}

\subsubsection{Thermal Test Protocols \& Apparatus}

The design of the thermal prototype includes the geometric features realized from modeling efforts, as well as prototype-specific features such as support brackets and a proxy absorber coating. The following discussion details the geometric and material choices specific to each component.

Support Shell. The shell contains the receiver hardware and is designed to be mechanically stiff to resist deflection during testing. In addition, the shell is mounted in a simple rotating frame to enable thermal testing over a range of receiver orientations, simulating collector tracking.

Absorber Tube. The absorber tube is fabricated from 316 stainless steel to enable high temperature testing $\left(650^{\circ} \mathrm{C}\right)$ without a dramatic loss in mechanical integrity. A commercial receiver for $650^{\circ} \mathrm{C}$ operation may require an Inconel absorber as is discussed in section 2.2 above, but for initial testing purposes, stainless steel offers comparable thermal conductivity and is significantly less expensive.

Absorber Tube Brackets. The absorber tube is suspended from the top seam of the shell by a set of three brackets. These brackets consist of a vertical support hanger that rides in a slotted member, which in turn is welded to the absorber. The slotted design of these brackets enable the lateral thermal expansion of the receiver tube relative to the support shell.

Glass. Borofloat 33 borosilicate glass was selected for its high heat tolerance and thermal shock resistance.

Absorber Coating. The purpose of our current thermal testing is to validate the modeled performance of the receiver geometry for increased efficiency at high operating temperatures. With the goal of geometry and performance validation, we are simulating a solar-selective coating by running thermal tests with a low-emissivity coating and optical tests with an absorptive coating. In conjunction we are developing an atmospherically stable solar selective coating that has demonstrated strong optical and thermal performance. Larger scale deposition techniques are currently under development. For realistic results from the thermal test, the absorber coating should have an emissivity close to that of existing solar selective coatings; an emissivity of $0.10-0.15$ can be achieved. ${ }^{\text {xvi }}$ After a search in Q3 for an appropriate coating (e.g. black 
DE-EE0005803

Advanced Low-Cost Receivers for Parabolic Troughs

Norwich Technologies

chrome, bare polished steel, transparent oxygen-barrier coatings, low emissivity paints, ceramic chrome finishes), which had a low emissivity and high temperature stability we used advanced aluminum paint products, LO/MIT 1 and LO/MIT 2, which were effective up to $650{ }^{\circ} \mathrm{C}$. The LO/MIT coatings have an emissivity measured to be $\sim 0.23-0.25$ depending upon the coating formulation and coating technique.

Our design accounts for thermal expansion as the absorber grows in cross section and length during thermal testing. At $650^{\circ} \mathrm{C}$, we expect the largest absorber tube $(3$ in $\mathrm{OD} x$ 72 in) to expand in cross section by 0.030 inch and in length by 0.80 inch. The lengthwise expansion ( 0.80 in) has been accounted for in our design through the addition of CALCARB rigid insulation as endcap assemblies.

\subsubsection{Thermal Test Design and Procedure}

The Thermal Performance Test is modeled on the benchmark procedure developed by NREL's Receiver Test Laboratory. ${ }^{\text {xvii }}$ In the test, a copper tube is inserted into the absorber tube and located using spacers as shown in Fig. 11. A pair of cartridge heaters is inserted from either end into the copper tube, such that the ends of the cartridge heaters meet in the middle of the HCE. Inconel spacers are used to locate the cartridge heaters within the copper tube. The high conductivity of the copper tube serves to spread the power from the cartridge heaters more evenly throughout the inside of the absorber tube. With a set of Variacs, the amount of heat put into the HCE can be controlled to reach steady state at the desired operating temperature. At steady state conditions, heat supplied balances heat lost to the surroundings through a combination of radiation and convection.

Fig. 11. Thermal Test setup (based on NREL thermal test)

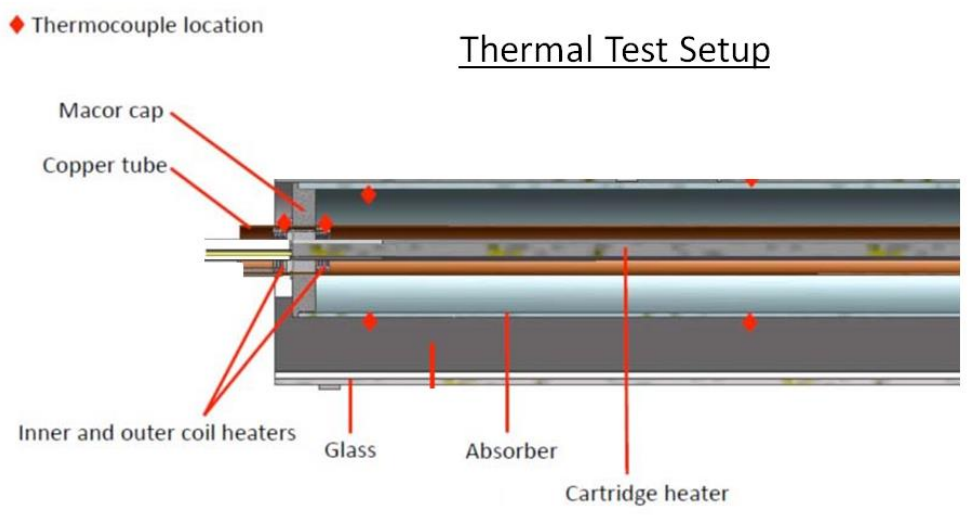

The NREL procedure involves the use of secondary heaters at each end of the receiver to control and measure end heat losses and to create adiabatic boundary conditions at the ends. Two coiled cable heaters (herein "coil heaters") are wound inside each end of the main heater assembly (a copper tube containing suspended cartridge heaters) and driven independently. The inner set of coil heaters is powered to maintain a flat temperature profile along the absorber tube, and the outer set of coil heaters maintain a flat temperature profile in the copper tube heater assembly (thermocouples are mounted near the inner and outer heaters to monitor the local temperature gradient). The inner 
coil heater powers thus represent end heat losses from the receiver body and are added to the cartridge heater powers to obtain a total receiver heat loss. The outer coil heaters serve to create an adiabatic boundary condition at the ends of the cartridge heater assemblies and so their powers are not included in the receiver heat loss calculation. The ends of the receiver are well insulated to reduce spurious heat losses. We take advantage of this boundary condition setup to shorten our test receiver to a $6 \mathrm{ft}$ length, which reduces the build cost and complexity without having end effects dominate.

To construct a heat loss vs. temperature curve, we incrementally increased the electrical power to the cartridge heaters until the absorber temperature approached each target temperature. As we approached the desired operating temperature, the power ramp rate was slowed and then held constant to ensure steady state operation at the nominal desired operating temperature. Steady state was confirmed by perturbation of heater powers and by idling at each target temperature for a minimum of 30 minutes. Absorber temperature was be deemed to have reached the set point once the thermocouples placed along the length averaged to the target operating temperature. All data was recorded using an Omega InstruNet system.

\subsection{Optical Test Protocol \& Apparatus, Optical Prototype, and Materials Selection (Tasks 1.4, 1.5, 1.7, and 2.2)}

\subsubsection{Optical Test Apparatus and Prototype}

We evaluated the optical performance of the SunTrap-A and SunTrap-B receiver geometries with an optical test unit of our own design. For the SunTrap receiver geometry, the optical performance of the receiver depends not only on glass transmittance and coating absorptance, but also on the receiver geometry and its relation to the collector. Thus, our optical test setup involved constructing a parabolic trough collector as well as a receiver. Due to constraints on the mirror support machining process, the collector was limited to a 60 in. mirror aperture width, which corresponds to a $30.4 \%$ scale (from a $5 \mathrm{~m}$ collector). The optical test receiver unit is scaled accordingly, with absorber size adjusted to the closest available stock size.

\subsection{Optical Test Apparatus}

Fig. 12 illustrates the optical test hardware which comprises:

Mirror Assembly. Commercially sourcing a custom-shaped collector of the necessary accuracy proved impractical, and so Creare designed a means of creating an accurate parabolic mirror by conforming a mirrored surface to a set of precision-machined supports.
Fig. 12. Optical test stand, including collector on tilting base (a precision optical table on pillow blocks) and receiver connected to fluid loop. Not shown: cart with reservoir tank, circulating pump, and data acquisition system. A pyranometer is mounted to the optical table so that it is aimed along with the collector.

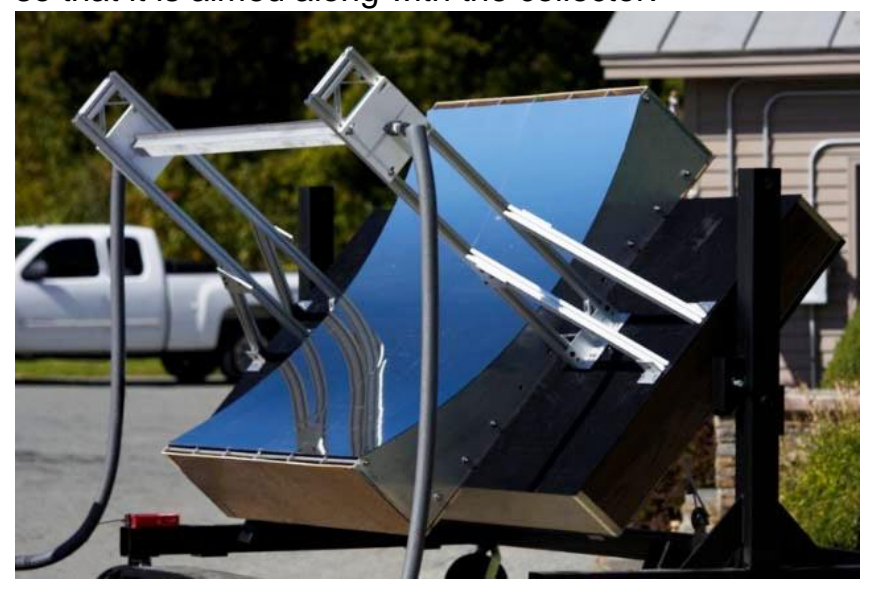


DE-EE0005803

Advanced Low-Cost Receivers for Parabolic Troughs

Norwich Technologies

The mirror assembly comprises a number of custom CNC machined struts, the mirror surface, and locking tabs. The custom machined struts contain the parabolic profile (machined relative to the back edge), and a set of holes (not shown) for easy mounting to the optical table. The mirror surface is a flexible sheet of polycarbonate which is covered with a highly reflective coating (ReflecTech solar mirror film) using standard roll-to-roll processing methods. The mirror sheet is made to conform to the strut profiles without localized distortions by use of an adhesive bond.

Optical Table. We selected an optical table to provide a stiff, ultra-flat surface on which to assemble the mirror. The array of table mounting holes serves to properly position struts relative to one another and lock them in place.

Receiver Hardware. The receiver hardware comprises the receiver, receiver frame, and receiver locating bracket. These fix the receiver relative to the mirror and enable fine adjustment. The receiver is approximately $20 \mathrm{~cm}$ longer than the mirror on each side. This additional $20 \mathrm{~cm}$ will allow all of the reflected light from the mirror to be incident upon the receiver throughout the span of the optical test, as well as avoiding shadowing of the mirror by the receiver support hardware.

Mirror Alignment Adjustment. To avoid the complexity and inaccuracy of including a tracker and drive in the optical test stand, the collector was aligned along an E-W axis and tilted to match the elevation angle of the sun at solar noon. With a simple cableand-turnbuckle system, we were able to align the collector to the solar elevation angle at solar noon each day and visually confirm that concentrated light pattern was centered on the absorber tube at the start of each test.

Absorber Coating. For the optical test, the infrared emittance of the absorber tube is not significant (due to the low temperatures and high concentration ratio of the collector), and the only major consideration is that the absorber tube has high solar absorptivity. Our selected coating, Zynolyte black stove paint, is used in heat flux sensors for concentrated photovoltaic applications, and has a solar absorptivity of approximately 0.96 as reported in the literature and measured at Dartmouth College. ${ }^{\text {xiii }}$

\subsubsection{Optical Test Protocol}

Our optical test was originally designed to measure the temperature difference between the inlet and the outlet of the receiver tube, using a heat exchanger to reject heat from the loop and maintain an inlet temperature at near-ambient. The flow rate of the water loop would be adjusted to achieve a reasonable temperature rise between the inlet and outlet of the receiver while maintaining an average receiver temperature that is within a few degrees of the ambient temperature to minimize the thermal losses from the receiver. However, subsequent analysis revealed problems with this technique.

For the increase in temperature across a 2-m receiver tube to be large enough to be measured accurately $(\sim 5 \mathrm{~K})$, the fluid velocity must be relatively slow. When the fluid velocity is slow, then the heat transfer coefficient from the absorber tube to the fluid is low, resulting in an increase in the temperature of the absorber tube ( $80 \mathrm{~K}$ above fluid temperature). The absorber tube temperature becomes large enough such that, even if the fluid temperature is at ambient, the heat loss cannot be said to be small compared to the radiation input, and the optical efficiency cannot be accurately calculated. It was 
DE-EE0005803

Advanced Low-Cost Receivers for Parabolic Troughs

Norwich Technologies

desirable to revise the test concept rather than incurring the expense of constructing a much longer collector.

To resolve these issues, we designed a novel optical test in which fluid is circulated from an insulated reservoir and accumulated thermal energy is measured by monitoring the temperature of fluid (water) within the reservoir tank. With this technique, flow rate through the receiver can be arbitrarily high so that the absorber temperature is kept at near-ambient, minimizing errors due to heat loss. The increase in temperature of the fluid in the storage tank can be easily and accurately measured over the course of the experiment. A pyranometer measures insolation and enables the calculation of a system optical efficiency.

To account for thermal losses in the overall test setup, we incorporated an electrical heater in line with the receiver as an alternate means of heating the water. The water temperature rise with a known heater power (approximately equal to the power expected from the sun when the system is illuminated) over the expected on-sun test period is then be measured, providing an accurate estimate of the effective thermal mass and thermal losses in the system.

Scaling down the optical test mirror and receiver increases the requisite manufacturing and positioning tolerances. The following analysis establishes the necessary tolerances for the $30 \%$ scale optical test and discusses how they were met.

\subsubsection{Optical Prototype Alignment}

Since the sun elevation in Vermont is low compared to a typical solar plant location, it is desirable to perform the optical test around solar noon. For a twenty minute time span about solar noon, the solar elevation changes less than $0.1^{\circ}$ while the solar azimuth changes approximately $11^{\circ}$. Since the solar elevation changes little near to solar noon, we oriented the mirror along an E-W axis and fixed its tilt angle for the duration of the test. Analysis of mirror error and optical efficiency for various tracking errors indicates that it is required to match the elevation angle of the sun to within less than $0.2^{\circ}$. Final alignment at the start of each test was performed visually by centering the concentrated solar line on the absorber, which made sub-degree corrections readily achievable.

E-W alignment also minimizes cosine losses during the experiment. In a typical N-S trough system, cosine losses will stem from the solar elevation angle. In this scenario, at solar noon in August, the losses are 12\%. Testing into the Fall months would further increase these losses. In comparison, the cosine losses in this optical test are due to the solar azimuthal angle and are $\sim 2 \%$. To maximize the active mirror surface area when the sun's azimuthal angle is not exactly $180^{\circ}$, the receiver was made longer than the mirror. Making the receiver longer than the mirror allows for the full length of the mirror to reflect onto the receiver for a wide sweep of azimuthal angles (covering roughly 30 minutes before and after solar noon), as well as eliminating shading effects from the receiver support frame. Full utilization of the mirror enables straightforward calculation of the concentrated flux on the receiver. Since the receiver tube is at nearambient temperatures, the heat loss error due to this additional length is negligible. It is also important to quantify how accurately the mirror needs to be aligned along the E-W axis and how horizontal (well-leveled) the system must be. Fig. 13 shows a 
contour plot of optical efficiency for variations in solar elevation angle and E-W alignment error for an optical error of $6 \mathrm{mRad}$. The sun position is assumed to be at

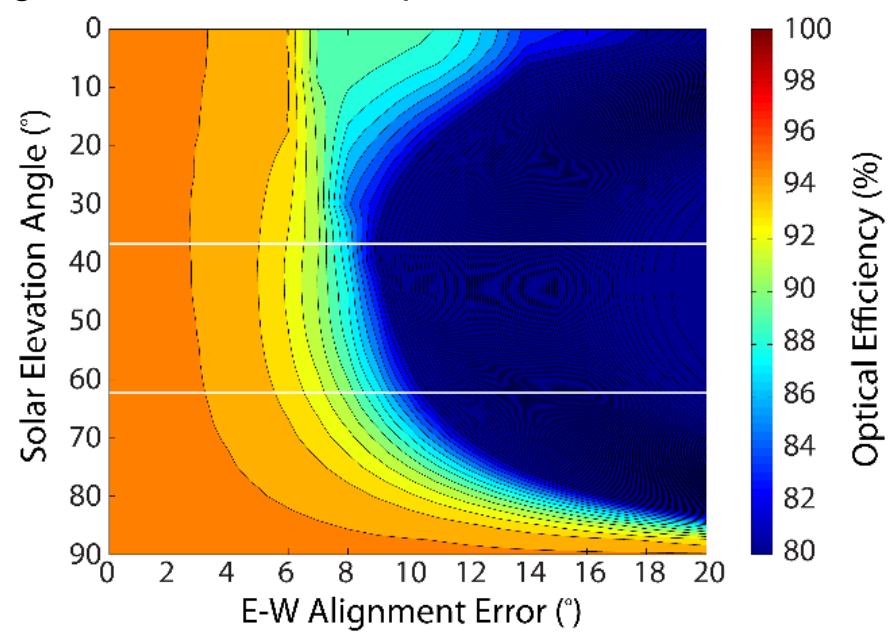

Fig. 13. Contour plot of optical geometric efficiency for variations in E-W alignment error and sun elevation angle for a $6 \mathrm{mrad}$ "total" error. Area between the white solid lines show the extent of sun elevation that occurs at solar noon between August and October in Norwich, VT. The asymmetry about sun elevation angle $=45^{\circ}$ is due to cosine effect and length of the receiver versus the mirror. "Bunching" of contour lines at near $0^{\circ}$ sun elevation angle is due to insufficient resolution of points along the trough.

solar noon. The required accuracy depends on mirror error and sun elevation (altitude). The E-W alignment (azimuth angle) is not as critical as altitude angle accuracy; $2^{\circ}$ is tolerable. The accuracy of mirror alignment parallel to the horizontal (i.e., how well the mirror is leveled) has a similar relationship; less than $2^{\circ}$ error is tolerable. Both $\mathrm{E}-\mathrm{W}$ alignment and leveling were easily achieved in the field. E-W alignment was achieved by putting the optical table parallel to a E-W compass line and confirmed with a plumbline shadow at solar noon (casting a line directly solar south); leveling was done by adjusting the height of one end of the collector base with a long level placed along the optical table surface.

For receiver positioning, it is desirable to be able to align the receiver to the mirror focal point to within $1 \mathrm{~mm}$ accuracy. As the mirror error gets smaller (e.g., $3 \mathrm{mrad}$ ), the alignment tolerance increases (e.g., $2 \mathrm{~mm}$ ). In practice, this adjustment was the most difficult to perform to the requisite accuracy due to the uncertainties of field alignment and the actual focal length of the collector.

\subsection{Optical and Thermal Testing, Model Refinement \& Validation, and Second- Generation Commercial Prototype (Tasks 3.1 and 3.2)}

The test results from our first-generation optical and thermal prototypes were highly successful. The primary objectives of these tests were to demonstrate the operational principles of our novel SunTrap receiver in accordance with the FOA's performance requirements and validate the underlying optical and thermal models. The strong correspondence between measured and predicted values clearly demonstrates we have effectively quantified the important underlying physical mechanisms. In terms of thermal performance, this means our models capture the radiative, convective, and conductive loss mechanisms including the thermal loss pathways existent in a real physical system. In terms of optical performance, this means our models accurately account for optical errors in the system and properly treat the propagation of optical energy including accounting for the transmissive and absorptive properties of the materials involved. 
DE-EE0005803

Advanced Low-Cost Receivers for Parabolic Troughs

Norwich Technologies

\subsubsection{Thermal Results}

The thermal results demonstrate the ability to accurately capture and model the heat loss from the SunTrap prototype receivers and demonstrate the ability to achieve a thermal efficiency of $>90 \%$ as required by the FOA. This section details the calculations of experimental heat losses at a given temperature and how the uncertainties in these values is determined. The new Fluent model is described and the uncertainties in the model, associated with material properties are characterized. A comparison between the prototype experimental and modeled heat loss characteristics is made. Using lessons learned from the prototype results, proposed changes are suggested and analyzed for a secondgeneration "commercial receiver". The thermal efficiency

Fig. 14. Absorber and copper tube temperatures, and heat powers during test of SunTrap-B prototype.

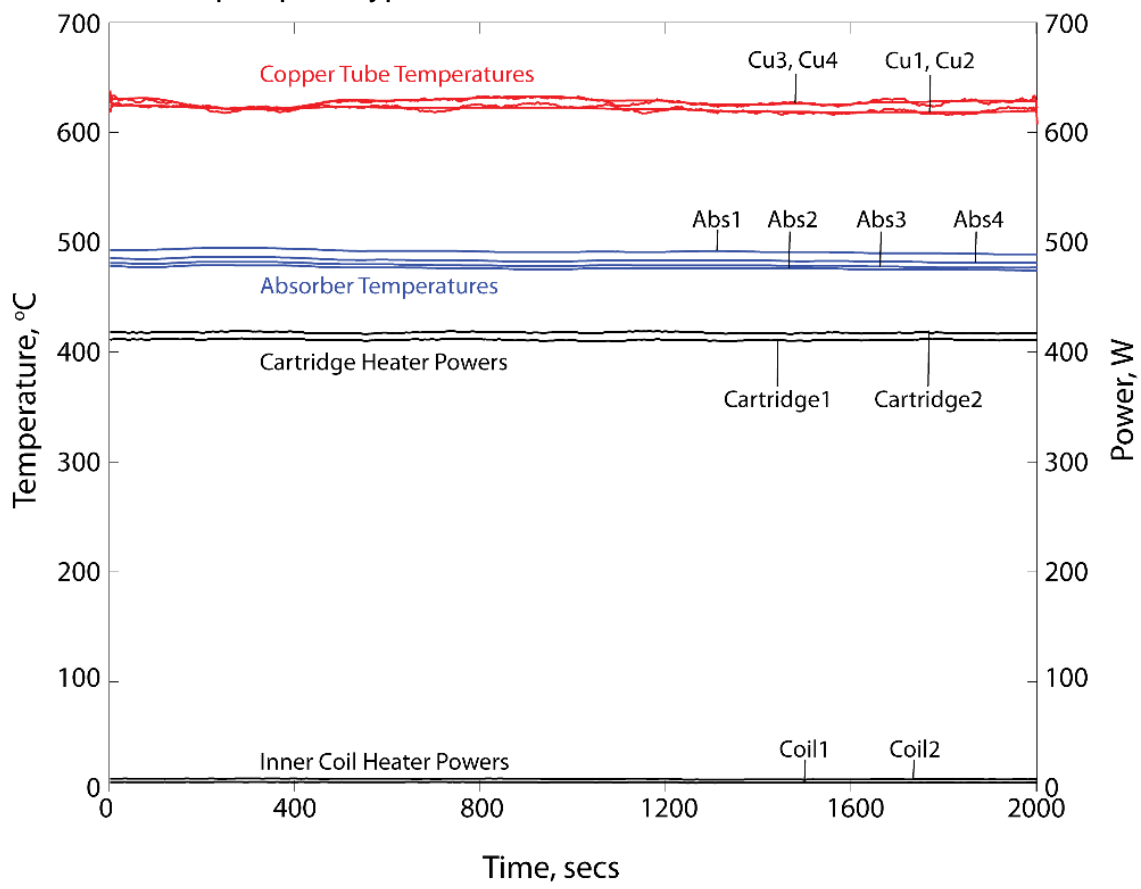
of a solar plant for this commercial receiver is calculated and compared to the FOA requirements.

Example Result: SunTrap-B Prototype at $\sim \mathbf{4 8 0} \stackrel{\circ}{ }$ C. Fig. $\mathbf{1 4}$ shows the recorded powers and temperatures gathered for one heat loss test for the SunTrap-B prototype receiver. Depicted are the absorber temperatures (Abs1-Abs4), copper tube temperatures (Cu1-Cu4), heater powers (Cartridge1, Cartridge2, Coil1,Coil2) during the 2000 second test period. The absorber and copper tube temperatures and heat powers vary little throughout the "steady-state" test. Cu4 and Cu2 closely match Cu3 and Cu1 respectively as the power generated by the outer coil heater power is varied in order to maintain an adiabatic boundary condition at the ends of the receiver.

The average absorber temperature, $A b s_{\text {ave }}$ is given by

$$
A b s_{\text {ave }}=\frac{A b s 1+A b s 2+A b s 3+A b s 4}{4},
$$

where $A b s 1, A b s 2, A b s 3, A b s 4$ are the average temperatures measured by thermocouples 1-4 respectively over the course of the test. The average absorber temperature is $481 \mathrm{C}$. The total heat loss $(\mathrm{W} / \mathrm{m})$ from the receiver is given by

Heat Loss $(W / m)=\frac{\text { Cartridge } 1+\text { Cartridge } 2+\text { Coil } 1+\operatorname{Coil2} 2+\frac{k \cdot A}{\Delta x}(C u 1-\text { Cu } 2)+\frac{k \cdot A}{\Delta x}(C u 3-C u 4)}{L_{H C E}}$, 
DE-EE0005803

Advanced Low-Cost Receivers for Parabolic Troughs

Norwich Technologies

where Cartridge 1 and Cartridge 2 are the average cartridge heater powers (W), Coil 1 and Coil2 are the average inner coil heater powers $(\mathrm{W}), k$ is the thermal conductivity of the copper $(\mathrm{W} / \mathrm{m}-\mathrm{K}), A$ is copper pipe's cross-sectional area $\left(\mathrm{m}^{2}\right), C u 1$, $\mathrm{Cu} 2, \mathrm{Cu} 3$ and $\mathrm{Cu} 4$ are the average temperatures of the copper tube near the ends of the prototype as shown in Figure P1, $\Delta x$ is the distance between $C u 1$ and $C u 2$, and $C u 3$ and $C u 4(\mathrm{~m}), L_{H C E}$ is the length of the prototype (m).

The majority of the thermal power is from the cartridge heaters. In this case, the power produced by the cartridge heaters are $406 \mathrm{~W}$ and $416 \mathrm{~W}$ and the power produced by the inner coil heaters are $7 \mathrm{~W}$ and $8 \mathrm{~W}$, giving the total power into the receiver as 827 W. In this case, since $C u 1$ and $C u 2$, and $C u 3$ and $C u 4$ are well matched, the last two terms in the foregoing equation contribute less than $3 \mathrm{~W} . L_{H C E}$ is $1.82 \mathrm{~m}$, resulting in a total heat loss of $460 \mathrm{~W}$.

Thermal Results: Uncertainty. For the prototype, the uncertainty in the measurements comes from variability in the measurements and the accuracy of the measurement instrumentation itself.

As shown in Fig. 14, little variability exists in any of the individual values throughout the course of the run. Since little variability exists in the power from the heaters throughout the run and the accuracy of each heat measurement is high $(\sim+/-0.5 \%)$ the uncertainty in the power is low. For example, for this experiment where the total power is $\sim 850 \mathrm{~W}$, the error in the power measurement is approximately $\pm 3 \mathrm{~W}$. For adiabatic conditions to exist at the end of the receiver tube, the copper temperatures at the inner and outer coil heaters must match. Since this temperature difference is usually low $\left(<3^{\circ} \mathrm{C}\right)$, the resulting heat loss is low and the impact of any errors in this measurement on the results is negligible. The error in the absorber tube measurement arises from the accuracy of the thermocouples (greatest of $2.2^{\circ} \mathrm{C}$ or $0.75 \%$ ) and the variability in the temperature readings between thermocouples. The variability in the temperatures measured by the thermocouples is not unexpected due to the difficulty in uniformly heating the tube. To account for this uncertainty, the standard deviation of the absorber temperatures is used to estimate this error. For the experiment shown in Fig. 14, the standard deviation between measurements is $4.9^{\circ} \mathrm{C}$ and the error in the thermocouple measurement is $3.6^{\circ} \mathrm{C}$ resulting in a combined error of $\pm 6.0^{\circ} \mathrm{C}$.

FLUENT Thermal Model. Thermal performance of the prototype receivers was determined by analyzing the results of radiative-loss, convective-loss, and conductiveloss models in ANSYS FLUENT. The developed thermal models are based on a threedimensional "short" section of the receiver with a constant temperature boundary condition at the external surface of the absorber tube in order to best model the prototype setup.

For the SunTrap-B receiver, the exposed surface area was coated with LO/MIT2 which had an estimated emissivity of $0.23( \pm 0.02)$ while the SunTrap-A receiver was coated with LO/MIT1 which has an estimated emissivity of $0.25( \pm 0.02)$. As measured by Dartmouth, the reflectance of the coating is relatively flat suggesting that the emissivity should not change (relative to the error bars) with temperature. These coatings were used as they have a known emissivity, and are stable in air at high temperatures. Future testing will incorporate atmospherically stable solar selective coatings such as the 
DE-EE0005803

Advanced Low-Cost Receivers for Parabolic Troughs

Norwich Technologies

coating developed by the Liu group at Dartmouth, to which Norwich Technologies has negotiated an exclusive world-wide license.

An additional refinement to the thermal modeling is an examination of the variability in heat loss that may arise due to uncertainties in various material properties. Using a wide range of Fluent modeling it is estimated that the combination of these errors contribute an error of approximately $\pm 4.5 \%$.

Thermal Results: SunTrap-A and SunTrap-B Prototype Data and Models. Fig. 15 shows the excellent correspondence for heat loss from the prototypes compared to the Fluent models for the SunTrap-A and SunTrap-B receivers. The errors in the prototype calculation are dominated by the error in the measurement of the temperature rather than power. The errors in heat loss are well within the vertical thickness of the error bars. For the Fluent model, the temperature is directly set and the heat loss is measured while for the prototype, the power input (heat loss) is directly set and the temperature is measured.

Fig. 15. Heat loss from the SunTrap-A (left) and SunTrap-B (right) prototypes vs Ansys Fluent model.
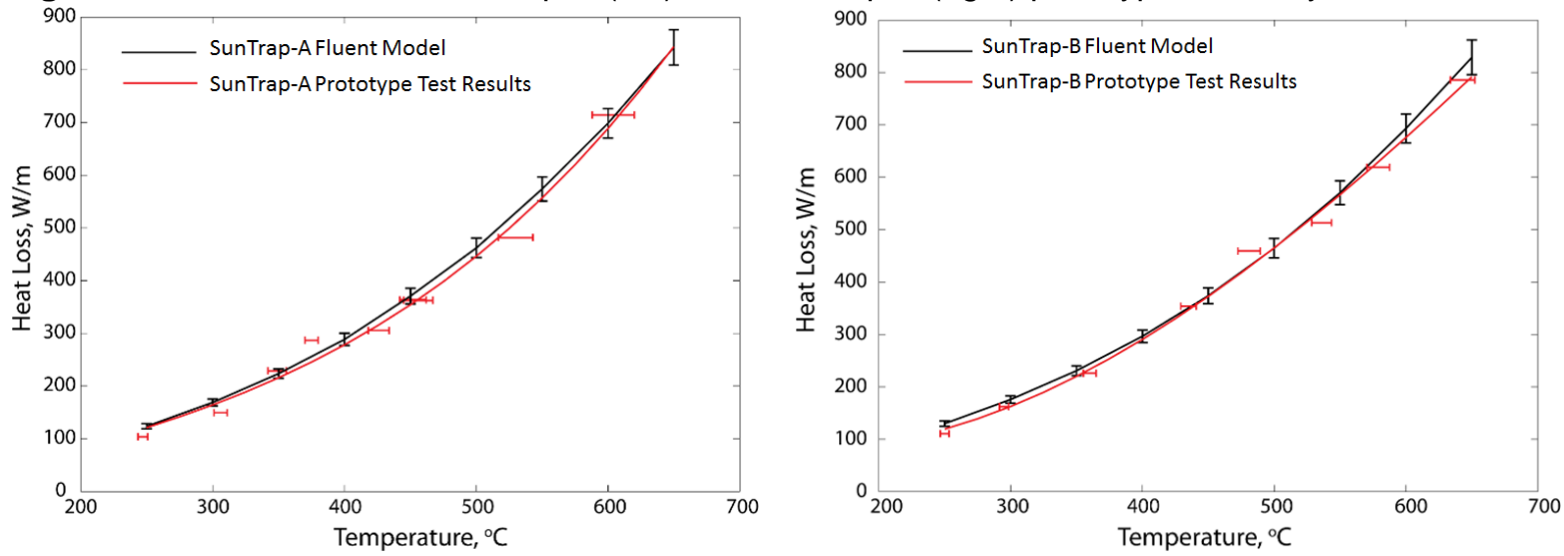

For a more direct comparison between prototype and model heat losses, the error in the temperature was converted to an error in heat loss (Fig. 16). In this case, the error in heat loss is calculated by assessing the heat loss at the error limits at each temperature using the curve of best fit. The difference in heat loss at the error limits temperature and the "best guess" temperature is combined with the heat loss from the heater and used as the error. It is clear that the Fluent model and the results from the Prototype are highly consistent with each other and are in general within the limits of uncertainty/error, thus demonstrating the ability to accurately model the system and account for the important underlying physical principles. Given the extent of our model development and refinement work and the effort that has been dedicated to an understanding of the physics of the heat loss mechanisms in the prototype, it is not surprising that the Fluent model to is able to accurately reproduce the prototype heat loss. 
DE-EE0005803

Advanced Low-Cost Receivers for Parabolic Troughs

Norwich Technologies

Fig. 16. Heat Loss from SunTrap-A (left) and SunTrap-B (right) prototypes compared to Ansys Fluent Model.
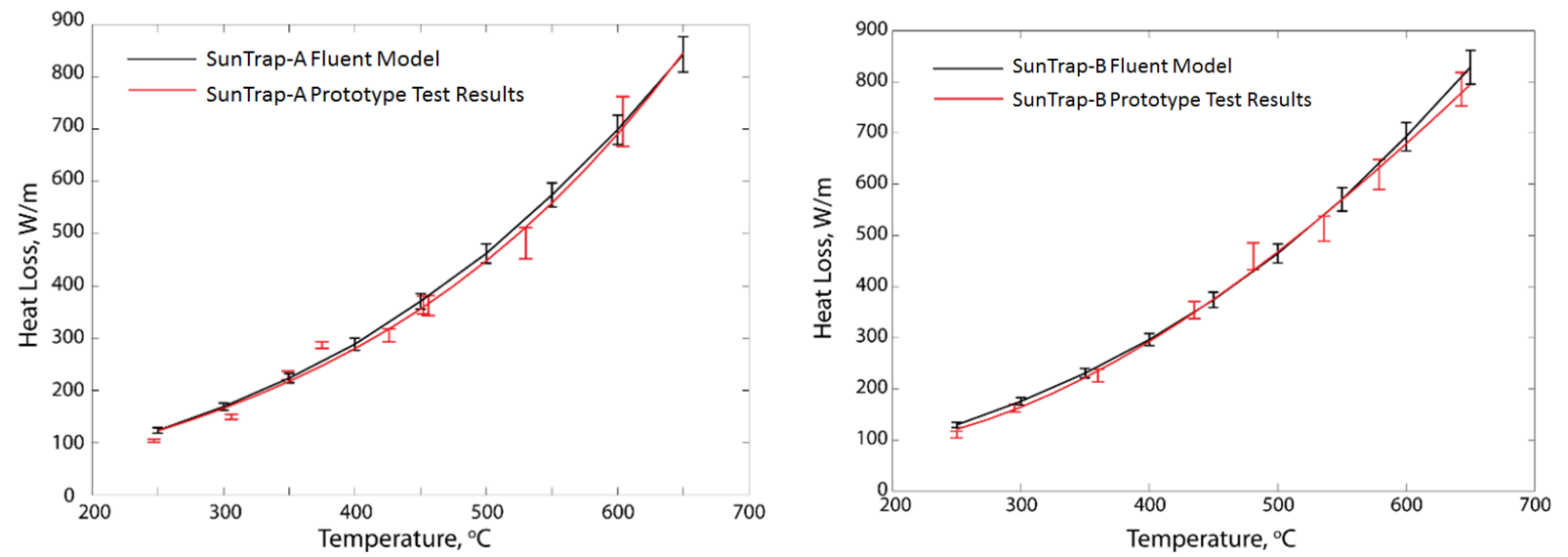

From Testing Prototype to Commercial Prototype. Our next-generation receiver, which we term the "Commercial prototype," will improve on our current prototype performance through the use of a new solar selective coating and the redesign of the internal insulation and support structure. The current coating was chosen as it has a known emissivity value and can be used to validate the modeling work. However, it has sub-ideal properties, e.g., an emissivity of 0.23 over 250-650 ${ }^{\circ} \mathrm{C}$ compared to the SOA, which is approximately 0.07 at $250^{\circ}$ $\mathrm{C}$ and 0.15 at $650^{\circ} \mathrm{C}$. Current work through NSF SBIR award Number IIP1315245 by the Liu group at Dartmouth and NT has developed a hightemperature,

Fig. 17. Comparison of heat loss from SunTrap first-generation prototypes, modeled Commercial prototypes, and Schott PTR70. Error bars removed for clarity.

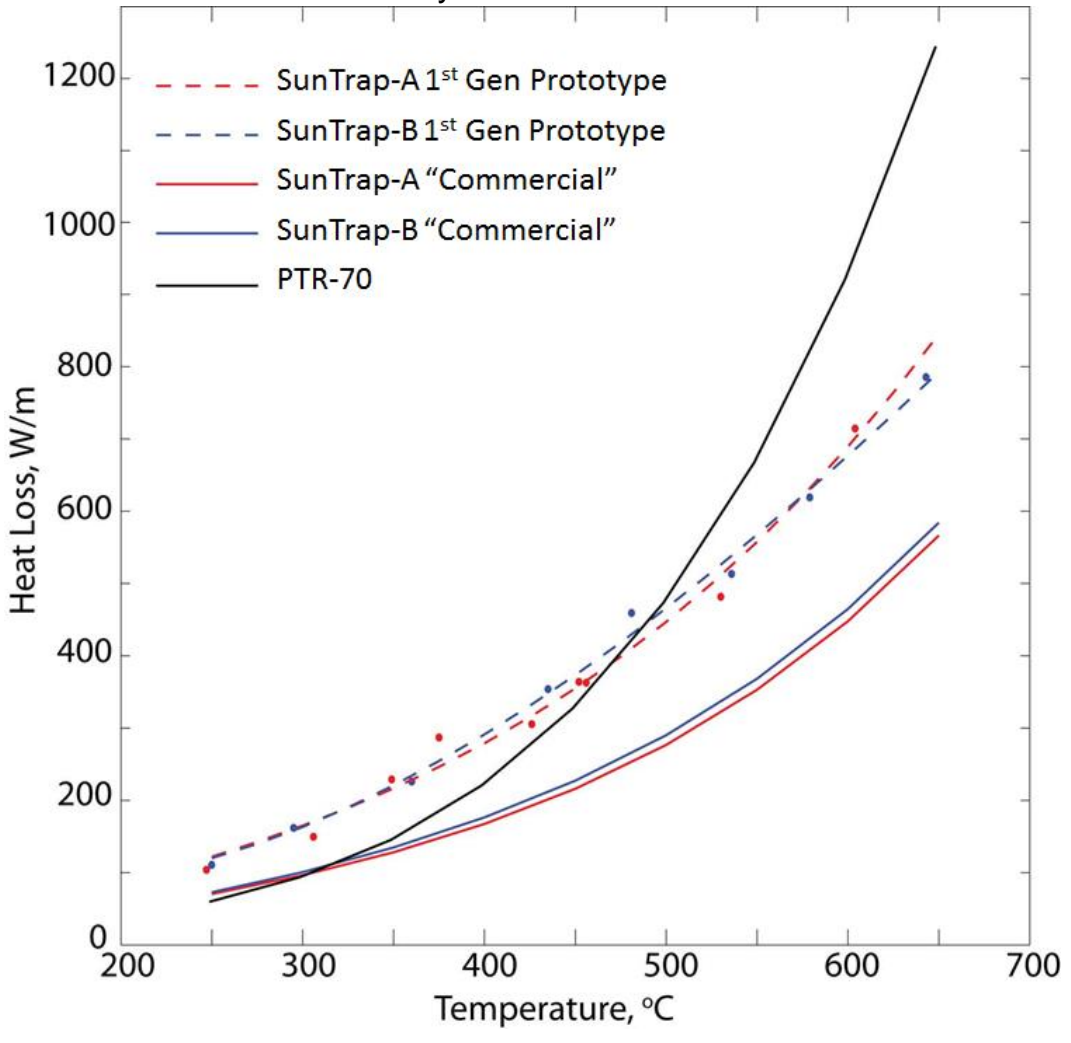

atmospherically stable, plasmonic nanochain solar selective coating which has similar optical and thermal properties to the coating used on SOA tubes (which require a vacuum). It is anticipated that large-scale deposition of this coating will be available in early 2014. 
DE-EE0005803

Advanced Low-Cost Receivers for Parabolic Troughs

Norwich Technologies

Regarding geometry, our tested prototype geometry was elected for simplicity in component acquisition and assembly but differs from the ideal geometry of a commercial receiver.

Fig. 17 shows a comparison of the heat loss from the PTR-70, the as-built prototypes and the expected heat loss from the Commercial prototype. The latter exhibits a 200 $\mathrm{W} / \mathrm{m}$ reduction in the heat loss at $650^{\circ} \mathrm{C}$ owing to the decreased coating emissivity and removal of prototype identified conductive heat loss paths. Both the as-built prototype and the Commercial have lower heat loss at high T than the current SOA.

For the first-generation prototype geometries, the exposed surface area of the SunTrap$A$ and SunTrap-B tubes are comparable. However, the absorber coating on the SunTrap-B tube has a lower emissivity $(0.23$ compared to 0.25$)$ than the SunTrap-A tube. Below $550 \stackrel{\circ}{ } \mathrm{C}$, where radiative losses are less dominant, the SunTrap-A tube outperforms the SunTrap-B prototype. Above $550^{\circ} \mathrm{C}$, the SunTrap-A tube has higher heat loss owing to the increased emissivity of the coating. Since the coating on the "commercial" models is the same, the SunTrap-A tube has lower heat loss than the SunTrap-B tube at all temperatures. Future work will require optimization of the receiver geometry to maximize performance and minimize cost with respect to the available mirror geometries and accuracies.

\section{Simulated Thermal Efficiency of Solar Field for Typical Daily Operation. NT} developed a novel solar field model in MATLAB, allowing us to study the simulated plant performance over modeled operating conditions. Operating conditions include the collector string temperature profile from inlet to outlet, the variable insolation during a typical day, tilt angle of the receiver and cosine effects at different times of the day. In these simulations, it was assumed that the inlet temperature was $350^{\circ} \mathrm{C}$, the outlet temperature was $650^{\circ} \mathrm{C}$ and the peak of the daily insolation was 1000 $\mathrm{W} / \mathrm{m}^{2}$. The solar field length was sized so that the peak daily mass flow rate was $6.22 \mathrm{~kg} / \mathrm{s}$. Cosine effects were calculated for a typical summer day in Phoenix, AZ.

To provide a comparison for a commercial installation, Fig. 18 compares the thermal efficiency our first- and second-generation SunTrap commercial prototypes with the PTR70. Optical efficiencies were estimated Fig. 18. Daily thermal efficiency for solar field running from $350-650^{\circ} \mathrm{C}$.

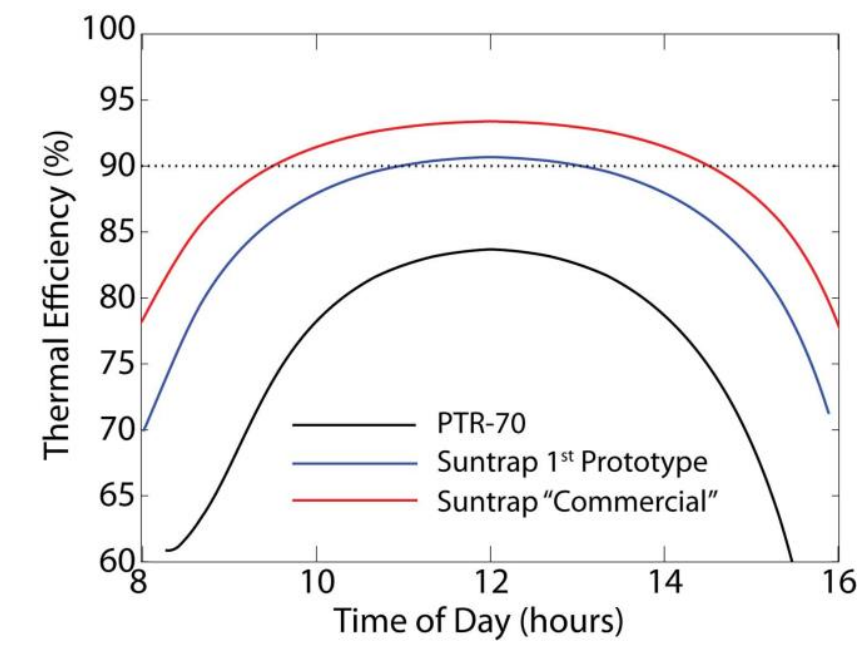
to be $\sim 80 \%$. Variations in optical efficiency (e.g., $\pm 5 \%$ ) do not make a significant difference to the thermal efficiencies $(<1 \%)$. It was assumed that the $\Delta \mathrm{T}$ between fluid and absorber tube was the same at all temperatures and flow velocities (derived from solar insolation). Analysis by ENEA for the Archimede solar plant suggests that as the solar insolation decreases (and subsequently the mass flow rate decreases), $\Delta T$ also decreases. Therefore, these results may overestimate the heat loss at lower insolation values. 
DE-EE0005803

Advanced Low-Cost Receivers for Parabolic Troughs

Norwich Technologies

The peak thermal efficiency for our first-generation prototype receiver assuming a $6.5 \mathrm{~m}$ mirror, delivering an exit temperature of $650^{\circ} \mathrm{C}$ is $90.4 \%$ for a solar field running from 350-650 ㄷ. The "commercial" second-generation prototype using a better coating and improvements in structural support design is calculated to further increase the thermal efficiency to $93 \%$ and has a thermal efficiency over 90\% from $\sim 9: 30$ to $\sim 14: 45$. The typical operating time at $650 \mathrm{C}$ is approximately $08: 45$ to $15: 15$ with an average thermal efficiency of $92.3 \%$.

The PTR-70 model includes a typical $5 \mathrm{~m}$ mirror and the thermal performance peaks at $83 \%$ at noon. Improvements in mirror design would enable the use of a $7 \mathrm{~m}$ mirror which has a similar optical efficiency to the $5 \mathrm{~m}$ mirror typically employed with the PTR-70. The increase in solar energy on the receiver increases the peak thermal efficiency by $\sim 5 \%$, to $\sim 88 \%$. The average thermal efficiency of the PTR70 between these times is $78.9 \%$ and $84.8 \%$ for the standard and "advanced mirror" systems respectively.

\subsubsection{Optical Results}

The optical results demonstrate a method to accurately characterize the optical efficiency of the prototype and mirror using a novel test procedure. The description of the testing procedure for publication is currently in preparation. The procedure for the calculation of the experimental optical efficiency is outlined, followed by a comparison between the SunTrap-A and SunTrap-B receivers. The performance of the prototype is then compared to estimated theoretical efficiencies developed using known material properties and ray tracing analysis. These results and new testing capabilities will facilitate future testing and analysis including solar selective coating performance, alternative geometries, fluid flow properties and at temperature testing.

\section{Optical Results: Calculation of Heat} Capacity of the System. In order to estimate the thermal capacity of the system an inline heater was used to produce a known power input into the optical prototype setup. In this case, the mirror was covered to remove the solar radiation input. The inline heater delivered a power of $1188 \mathrm{~W}$ over 2007 seconds producing a total heat input of 2.384

Fig. 19. Rise in temperature of fluid in reservoir over test period (red) and line of best fit (black). $95 \%$ confidence limits are shown in equation.

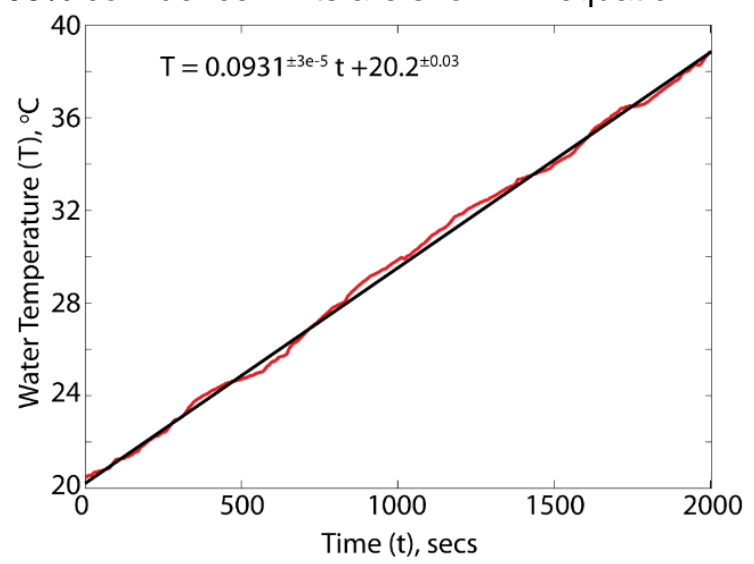
MJ. A total of $8 \mathrm{gal}$ water was used in the reservoir and circulated through the optical prototype at $\sim 16 \mathrm{gpm}$. The heat rise in the water in the reservoir over the test period is shown in Fig. 19.

The heat capacity of the system can be calculated from the equation, $m c=\frac{Q}{\Delta T}$. where $Q$ is the total heat input, $m c$ is the heat capacity and $\Delta T$ is the change in temperature. $\Delta T$ can be calculated as $0.0931^{ \pm 3 \times 10^{-5}} t$ where $t$ is the total testing time (2007 seconds), resulting in a thermal capacity of the system of $1.275^{ \pm 0.004} \times 10^{5} \mathrm{~J} / \mathrm{K}$. The calculated value also intrinsically includes any heat loss from the piping of water throughout the prototype. This thermal capacitance may be used for testing under solar radiation 
DE-EE0005803

Advanced Low-Cost Receivers for Parabolic Troughs

Norwich Technologies

assuming that the increase in heat loss owing to the raised temperature of the absorber tube under solar illumination is minimal. This value may be compared to the thermal capacitance of the water in the system at a mean temperature of $30^{\circ} \mathrm{C}$. At $30^{\circ} \mathrm{C}$, water has a mass of $996 \mathrm{~kg} / \mathrm{m}^{3}$ and a specific heat of $4.179 \mathrm{~kJ} / \mathrm{kg}-\mathrm{K}$ resulting in 8 gallons of water having a thermal capacity of $1.259 \times 10^{5} \mathrm{~J} / \mathrm{K}$. The similarity between the calculated and estimated values demonstrates that the thermal capacitance of the system is dominated by the water.

Optical Results: Results of Example Test-SunTrap-A Test 1. Test 1 was performed over 1,140 seconds, from 12:30 pm to 12:49 pm under partially cloudy skies. Fig. 2 (left) shows the irradiance profile over this time period. The average irradiance was $782^{ \pm 23} \mathrm{~W} / \mathrm{m}^{2}$. The error is due to the $\sim 3 \%$ uncertainty in the sensor measurement. Fig. 220 (right) shows the increase in water temperature over this time period. Eight gallons of water was stored in the water reservoir and circulated throughout the prototype. The increase in temperature was $0.01066^{ \pm 0.00005} \mathrm{~K} / \mathrm{s}$.

Fig. 20. Left: Solar irradiance over SunTrap-A test 1. Dotted black line shows average solar irradiance $\left(782 \mathrm{~W} / \mathrm{m}^{2}\right)$. Right: Increase in temperature of water in reservoir for SunTrap-A test 1. Solid black line shows fitted increase in temperature.
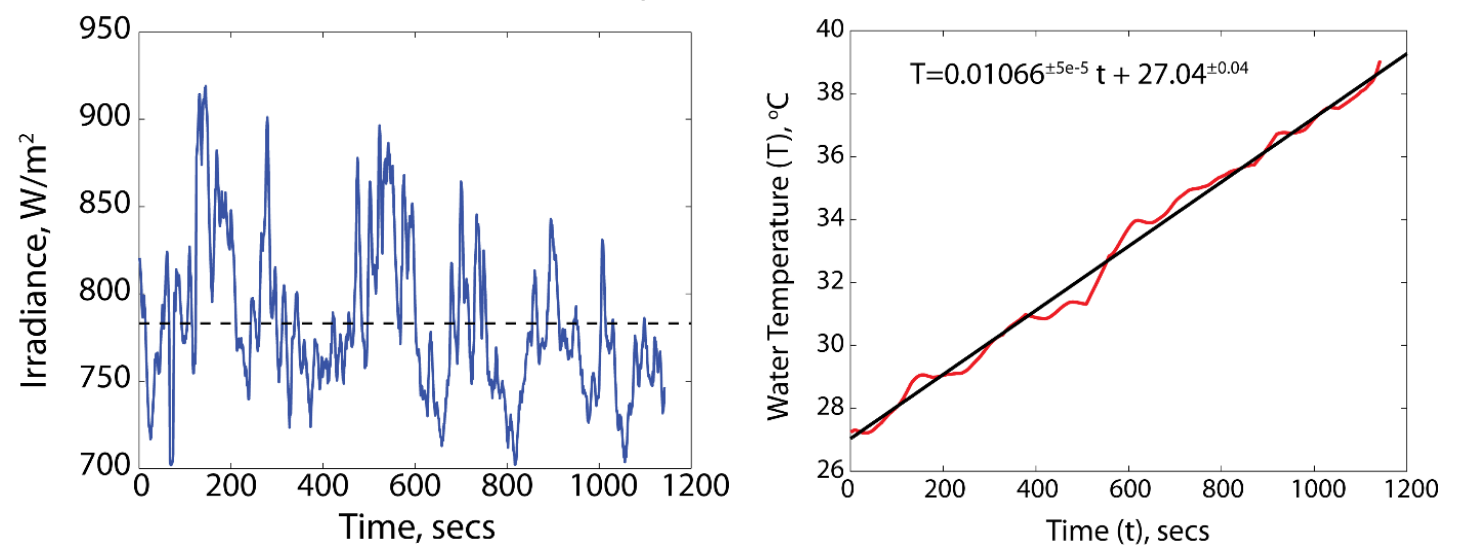

The solar energy that impinges upon the mirror over the course of the test is given by

$$
E_{\text {in }}=A \int_{0}^{t} I . d t=A \cdot I_{\text {ave }} t,
$$

where $E_{\text {in }}$ is the direct solar energy that falls upon the mirror, $A$ is the mirror area, $I$ is the instantaneous direct solar irradiance, $t$ is time and $I_{\text {ave }}$ is the average solar irradiance over the course of the test. The active mirror area is $2.20 \mathrm{~m}^{2}$. Therefore, for SunTrap-A test $1, E_{\text {in }}=1.96^{ \pm 0.058} \mathrm{MJ}$.

The energy that is transferred to the fluid is given by $E_{\text {out }}=m c \Delta T$, where $m c$ is the thermal capacitance of the system calculated and is $1.275^{ \pm 0.004} \times 10^{5} \mathrm{~J} / \mathrm{K} . \Delta T$ is the change in temperature of the water reservoir and is $0.01066^{ \pm 0.00005} \mathrm{~K} / \mathrm{s} \times 1140 \mathrm{~s}=$ $12.16^{ \pm 0.06} \mathrm{~K}$. Therefore, $E_{\text {out }}=1.55^{ \pm 0.013} \mathrm{MJ}$.

The total efficiency of the system is $\eta=\frac{E_{\text {out }}}{E_{\text {in }}}$. Since the heat loss is negligible compared to the heat input owing to the low temperature (and has been taken into account in the calculation of $m c$ ), this efficiency represents the optical efficiency of the system.

Therefore, for SunTrap-A test $1, \eta=79.1^{ \pm 3.0} \%$. 
DE-EE0005803

Advanced Low-Cost Receivers for Parabolic Troughs

Norwich Technologies

Optical Results: Results from Several Tests. Table 3 shows testing results for the SunTrap-A tube and Table 4 shows the results for the SunTrap-B tube.

Table 3. Calculation of optical efficiency for SunTrap-A absorber tube tests.

\begin{tabular}{|c|c|c|}
\hline & SunTrap-A Test 1 & SunTrap-A Test 2 \\
\hline Length of Test (s) & 1141 & 780 \\
\hline $\begin{array}{c}\text { Average Irradiance } \\
\text { (W/m } \mathbf{~}^{2} \text { ) }\end{array}$ & $782^{ \pm 23}$ & $883^{ \pm 26}$ \\
\hline Total Radiation Input (MJ) & $1.96^{ \pm 0.058}$ & $1.515^{ \pm 0.045}$ \\
\hline Water temp gradient (K/s) & $0.01066^{ \pm 0.00005}$ & $0.01206^{ \pm 0.00016}$ \\
\hline $\boldsymbol{\Delta T}$ (K) & $12.16^{ \pm 0.06}$ & $9.41^{ \pm 0.12}$ \\
\hline Energy output (MJ) & $1.55^{ \pm 0.013}$ & $1.20^{ \pm 0.015}$ \\
\hline Efficiency (\%) & $79.1^{ \pm 3.0}$ & $79.5^{ \pm 3.3}$ \\
\hline
\end{tabular}

Table 4. Calculation of optical efficiency for SunTrap-B tube tests. The error in SunTrap-B Test 3 is smaller than the others owing to the use of a more accurate pyranometer.

\begin{tabular}{|c|c|c|c|}
\hline & SunTrap-B Test 1 & SunTrap-B Test 2 & SunTrap-B Test 3 \\
\hline Length of Test (s) & 960 & 1500 & 1320 \\
\hline $\begin{array}{c}\text { Average Irradiance } \\
\left(\mathbf{W} / \mathbf{m}^{2}\right)\end{array}$ & $890^{ \pm 26}$ & $771^{ \pm 23}$ & $858^{ \pm 8}$ \\
\hline Total Radiation Input (MJ) & $1.88^{ \pm 0.056}$ & $2.54^{ \pm 0.016}$ & $2.49^{ \pm 0.025}$ \\
\hline Water temp gradient (K/s) & $0.01106^{ \pm 0.00009}$ & $0.00939^{ \pm 0.00008}$ & $0.01048^{ \pm 0.00004}$ \\
\hline $\mathbf{\Delta T}(\mathbf{K})$ & $10.6^{ \pm 0.09}$ & $14.1^{ \pm 0.11}$ & $13.8^{ \pm 0.05}$ \\
\hline Energy output (MJ) & $1.35^{ \pm 0.011}$ & $1.80^{ \pm 0.015}$ & $1.76^{ \pm 0.00 /}$ \\
\hline Efficiency (\%) & $71.8^{ \pm 3.1}$ & $70.9^{ \pm 3.1}$ & $70.7^{ \pm 1.1}$ \\
\hline
\end{tabular}

The optical efficiency of the SunTrap-B is less than the SunTrap-A owing to the difference in absorber surface area (see, Table 3, Table 4).

\section{Comparison to Theoretical Optical Efficiency}

\section{Absorber Coating}

Absorptivity. Fig. 21 shows

the wavelength dependence

of the black paint coating and the solar irradiation. The integrated absorptance of the coating across the solar spectrum is $95.9 \%$.

Mirror Optical Error. Fig. 22 (left) shows an example image of the reflected light from the absorber surface. This reflected light can be used to roughly approximate the profile of the reflected light on the absorber surface.
Fig. 21. Wavelength dependence of solar irradiance (blue) and coating absorptance (red).

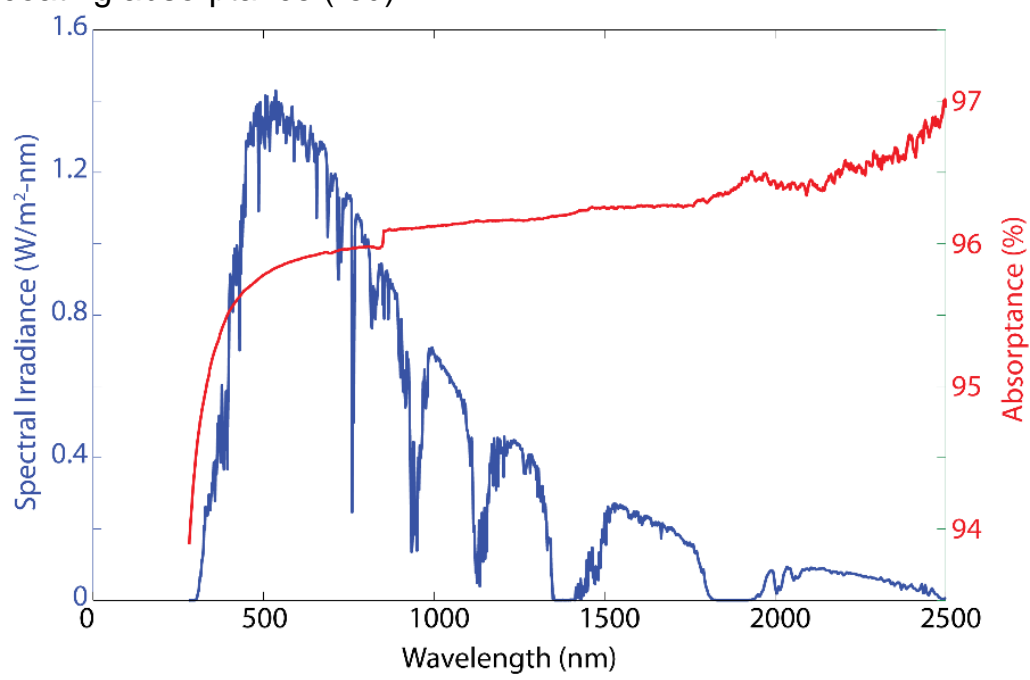

Fig. 22 (right) shows a comparison between the mean profile of the reflected light across the absorber tube and the profile on the absorber tube from the results of a ray tracing simulation using a mirror error of $3.5 \mathrm{mrad}$. Each profile is normalized to its peak 
DE-EE0005803

Advanced Low-Cost Receivers for Parabolic Troughs

Norwich Technologies

value. The profile of the calculated reflected light can be used as a proxy for the power that is incident on the receiver tube. For locations close to the center of the absorber tube (and the reflected beam), the profile reasonably mimics that of the simulated profile for an error of $3.5 \mathrm{mrad}$. However, further away, the profile breaks down with the intensity of reflected light being higher than the simulated profile. This difference is likely due to the inconsistency of the mirror error across the mirror surface and errors in the alignment of both the receiver tube, mirror and mirror surface.

Fig. 22. Left: Photograph image of beam of reflected light on the absorber surface. Right: Normalized profile of power for simulated profile (red) and profile calculated from image (blue).
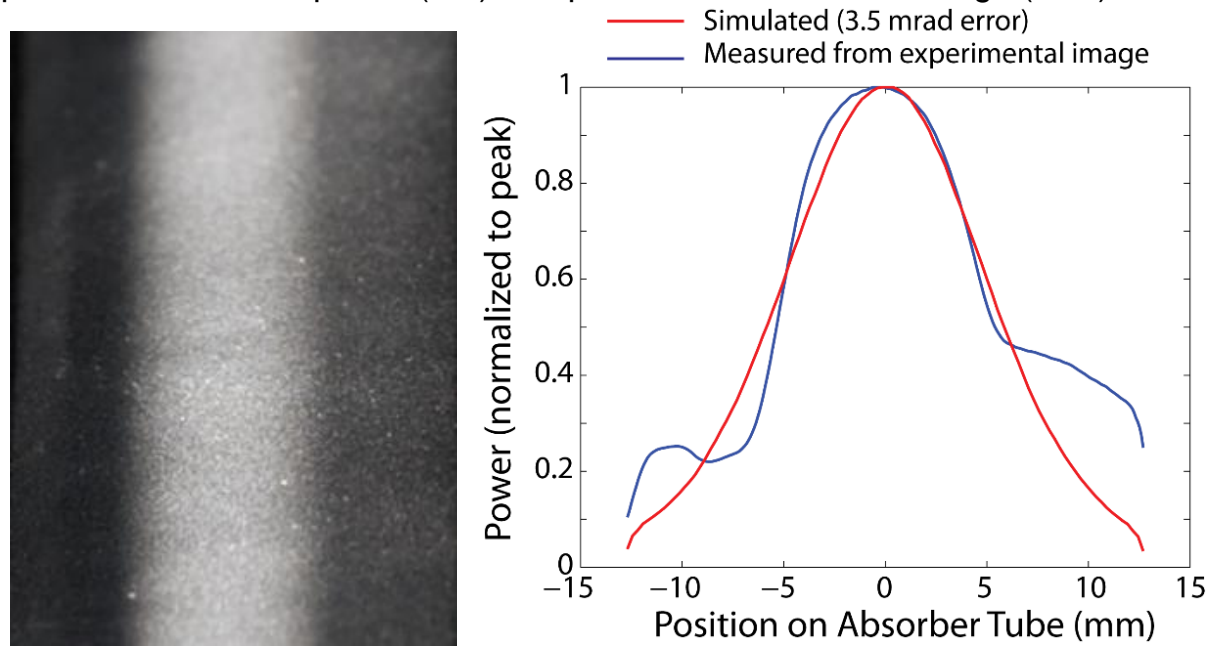

To estimate the mirror error and the geometric optical efficiency of the system, it is necessary to be able to equate the measured profile to a simulated profile. As a firstorder approximation, the standard deviation of the measured profile is compared to the standard deviation from the simulations (Fig. 23, left). The standard deviation of the

Fig. 23. Left: Comparison of standard deviation derived from image of absorber tube (black) with simulated variance of standard deviation and mirror error (red). Right: Simulated dependence of geometric optical efficiency with mirror error.


profile from the measured image is $\sim 5.75 \mathrm{~mm}$ which is consistent with the standard deviation using a simulated mirror error of $\sim 5.7 \mathrm{mrad}$. To estimate the mirror error and the geometric optical efficiency of the system, it is necessary to be able to equate the 
DE-EE0005803

Advanced Low-Cost Receivers for Parabolic Troughs

Norwich Technologies

measured profile to a simulated profile. As a first-order approximation, the standard deviation of the measured profile is compared to the standard deviation from the simulations (Fig. 23, left). The standard deviation of the profile from the measured image is $\sim 5.75 \mathrm{~mm}$ which is consistent with the standard deviation using a simulated mirror error of $\sim 5.7 \mathrm{mrad}$. Fig. 23 (right) shows how the mirror error affects the geometric optical efficiency of the system. For a simulated mirror error of $5.7 \mathrm{mrad}$ which is roughly equivalent to the optical prototype the geometric optical efficiency is $\sim 92 \%$. Future work will include a more accurate calculation of the radiation profile at the focal line.

Estimate of Theoretical Optical Efficiency. The theoretical optical efficiency may be estimated as

$$
\eta_{\text {opt }}=\eta_{\text {get }} \cdot \alpha \cdot R_{\text {mirror }} \cdot R_{\text {dirrt }} \cdot \cos (\theta),
$$

where $\eta_{\text {opt }}$ is the optical efficiency, $\eta_{g e o}$ is the geometric efficiency due to the error of the mirror, $\alpha$ is the absorptivity of the coating, $R_{\text {mirror }}$ is the nominal reflectivity of the $3 \mathrm{M}$ mirror reflective film, $R_{\text {dirt }}$ is a reduction in the mirror reflectivity due to dirt and wear and $\cos (\theta)$ represents the losses due to the cosine effects. Since the testing is done at near local noon, in this setup the reduction in irradiance due to cosine effect is negligible. The maximum off-incidence angle is approximately $10^{\circ}$ which gives a reduction in irradiance of $1.5 \%$. Most of the test is undertaken when the cosine angle is less than $10^{\circ}$ resulting in a loss due to cosine effect of $<1.5 \%$.

For this mirror, $\eta_{\text {geo }} \approx 0.92, \alpha \approx 0.96, R_{\text {mirror }} \approx 0.94$ and $\cos (\theta) \approx 0.99$. $R_{\text {dirt }}$ cannot be directly calculated but is expected to be approximately 0.95 . Therefore $\eta_{\text {opt }} \approx 0.781$ or $78.1 \%$. This value is consistent with the measured value of $79.3^{ \pm 3.0} \%$. It is probable that due to errors in the calculation of both the geometric efficiency and the reduction in reflectivity due to dirt that the theoretical efficiency may vary by up to $5 \%$. Future efforts will include work to reduce this uncertainty by maintaining and characterizing the state of the reflective surface and more accurately measuring the geometric efficiency.

Optical Results: Conclusions from Tests. The close correspondence between the optical test experimental data and estimated theoretical efficiency validates our understanding of the optical physics. In this experiment, the largest source of uncertainties comes from the calculation of the geometric efficiency and the reduction in reflectivity due to dirt. For this prototype, the mirror was developed for testing purposes and does not reflect the performance of a commercial mirror. It is anticipated that a commercial mirror would have a geometric efficiency of $\sim 4-5 \%$ higher.

The testing procedure and test setup developed in this project will be invaluable to future testing efforts. The new testing procedure has been validated and provides a simple, efficient and accurate method of characterizing the optical performance of the mirror and receiver system. It is anticipated that these testing procedure will benefit the parabolic trough solar thermal community and a manuscript is in preparation. The testing procedure may be adapted to higher temperatures in order to determine the thermal and overall efficiency of the prototype.

In addition, the results detailed in this report will provide a benchmark for future studies. For example, the solar absorptance of the new solar selective coating developed in NSF 
DE-EE0005803

Advanced Low-Cost Receivers for Parabolic Troughs

Norwich Technologies

SBIR Award number IIP-1315245 will be tested in the spring. The effect on optical performance of various glasses and the effectiveness of various AR coatings will also be tested.

The test setup also allows for the characterization of the fluid flow heat transfer properties. For example, water at near ambient temperature flowing through the optical prototype receiver has surprisingly similar heat transfer properties to molten salt at prototypical operating temperatures. The range of Prandtl numbers for solar salt from $300-600{ }^{\circ} \mathrm{C}$ is similar to that of water from $20-50^{\circ} \mathrm{C}$. The Reynolds number of the flow can be altered so that the Reynolds number of water in the optical test matches that of solar salt in a prototypical commercial plant. By measuring the temperature of the absorber tube compared to the fluid temperature it will be possible to calculate the relative heat transfer coefficients of SunTrap prototype receivers.

\subsection{Company and Marketing Development (Task 2.1)}

NT has established and continues to develop key technical and business relationships and internal resources. In addition the present SunShot project has established strong efficient collaborative working relationships with Creare Inc. and Ansys Inc.

\section{CONCLUSIONS}

In 12 months, we have completed an aggressive schedule to design, build and test a laboratory scale version of our low cost high performance trough receiver. By leveraging our technical and project management expertise, we have been able to create significant value on a relatively limited Department of Energy budget satisfying our milestones and go/no-go decision points. We are currently well positioned to proceed to the next stage of development.

Our future technical development work is focused on building on the highly successful results obtained during our SunShot grant. The specific development areas include (1) model, design, build and test the second generation of our novel SunTrap receiver, (2) integration of the second generation receiver with a low cost high performance cooptimized mirror collector, and (3) continuation of our successful solar selective absorber work currently funded by an NSF SBIR. 
DE-EE0005803

Advanced Low-Cost Receivers for Parabolic Troughs

Norwich Technologies

\section{REFERENCES}

' Burkholder F, Kutscher C. "Heat Loss Testing of Schott's 2008 PTR70 Parabolic Trough Receiver" (NREL/TP-550-45633): NREL, 2009.

ii Kutscher C, et al. "Line-Focus Solar Power Plant Cost Reduction Plan: NREL Milestone Report", 2010.

iii Mahoney R. "Trough Technology-Heat Collector Element (HCE) Solar Selective Absorbers" (Trough Workshop, ASES 2000): Sandia National Laboratories, 2000.

iv Kutscher, 2010.

${ }^{v}$ Gnadig T., et. Al., "SCHOTT's $4^{\text {th }}$ Generation Receiver - Getting ready for higher operation temperatures," SolarPACES 2013, Las Vegas, NV

${ }^{v i}$ Archimede Solar Energy, Receiver Tube - HEMS11, http://www.archimedesolarenergy.it/

solar_receiver_tube_for_thermodynamic_power_stations_hems $08 . \mathrm{html}$

vii Gnadig T., 2013

viii Burkholder and Kutscher 2009.

${ }^{i x}$ Kutscher et al. 2010.

${ }^{x}$ Khalsa SS and Ho C. "Radiation Boundary Conditions for Computational Fluid Dynamics Models of High-Temperature Cavity Receivers." J. Solar Energy Engineering-Trans ASME.

${ }^{x i}$ Rubbia 2001.

xii Rubbia 2001.

xiii Archimede Solar Energy, HEMS11 - DETAILED SPECIFICATIONS, http://www.archimedesolarenergy.it/hems11_detailed_specifications.htm

${ }^{x i v}$ R. Moore, M. Vernon, C. K. Ho, N. P. Siegel, and G. J. Kolb., "Design Considerations for Concentrating Solar Power Tower Systems Employing Molten Salt," Sandia Report, SAND2010-6978, 2010

${ }^{x v}$ Kutscher, 2010

xvi Burkholder and Kutscher 2009.

xvii Burkholder and Kutscher 2009.

xviii Ballestrín J, S Ulmer, A Morales, A Barnes, L.W Langley, M Rodríguez, Systematic error in the measurement of very high solar irradiance, Solar Energy Materials and Solar Cells, Volume 80, Issue 3, November 2003, 375-381. ISSN 0927-0248, at http://dx.doi.org/10.1016/j.solmat.2003.08.014. 